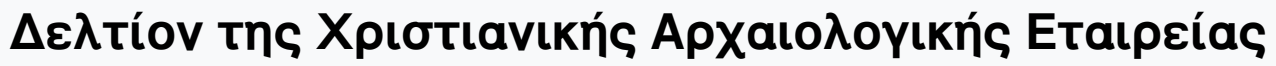

Tó 15 (1991)

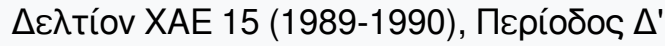

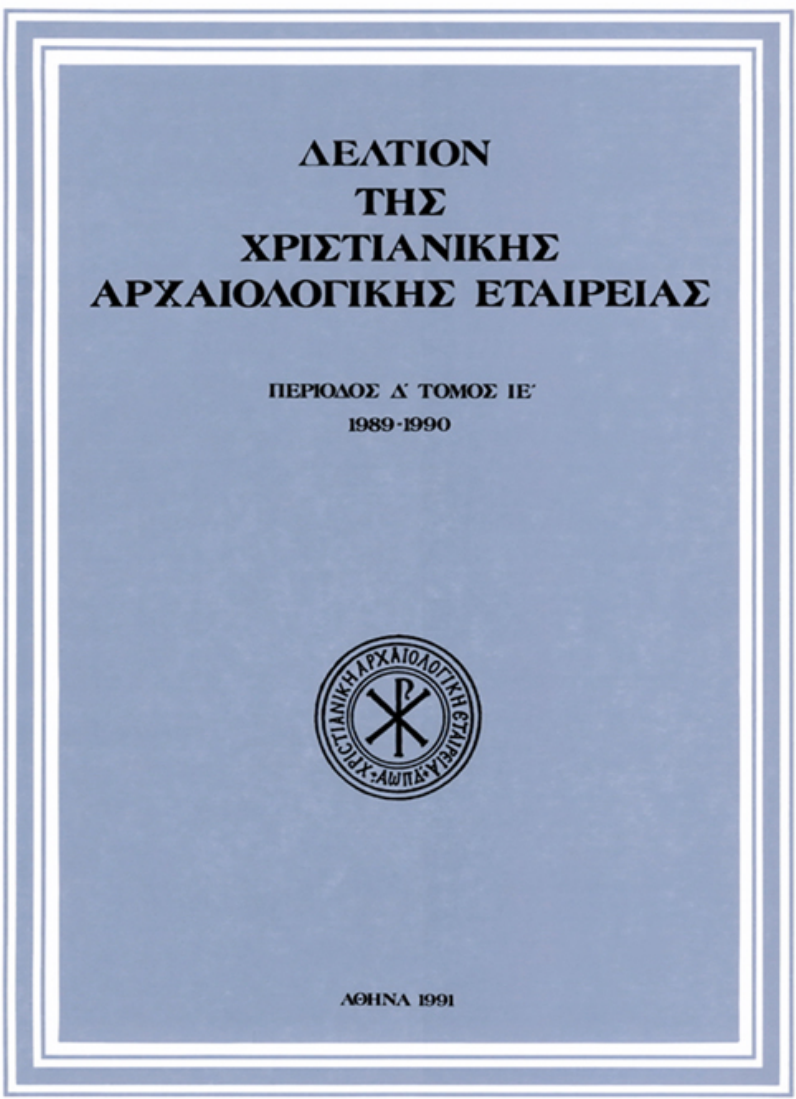

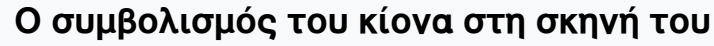

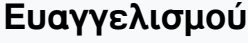

Hélène PAPASTAVROU

doi: $10.12681 /$ dchae.1039

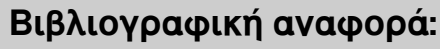

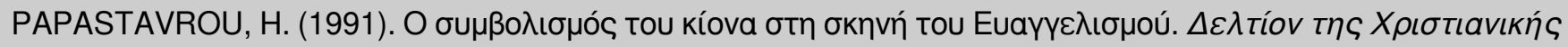

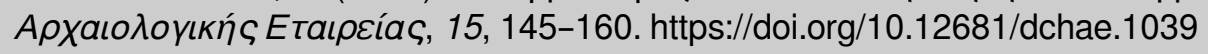




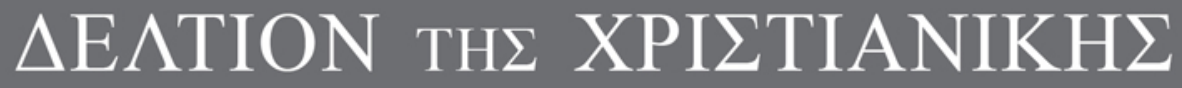 APXAIO $\Lambda$ OГIKH $\Sigma$ ETAIPEIA $\Sigma$}

Le symbolisme de la colonne dans la scène de l'Annonciation

Hélène PAPASTAVROU

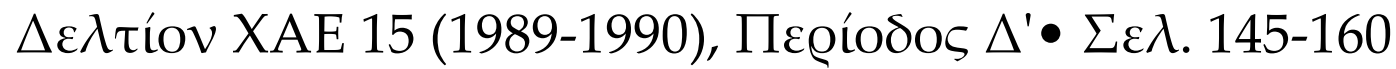
A@HNA 1991 


\section{Hélène Papastavrou}

\section{LE SYMBOLISME DE LA COLONNE DANS LA SCÈNE DE L'ANNONCIATION ${ }^{1}$}

Depuis l'antiquité, dans l'histoire de la civilisation, le motif de la colonne est employé fréquemment comme symbole politique ou religieux. On connaît les exemples de l'obélisque égyptien ou de la colonne votive de l'antiquité gréco-romaine. L'usage de cet élément a été légué à l'art paléochrétien et de l'époque médiévale. Souvent, son rôle dans l'art du moyen âge peut être symbolique, lorsque sa présence n'a pas une raison d'être fonctionnelle. Le présent travail est consacré à l'examen du symbolisme de la colonne dans certaines Annonciations occidentales et byzantines. Dans ce but et dans le cadre de la problématique que ce motif iconographique pose, nous allons plus particulièrement évoquer la manière dont les textes patristiques grecs et latins ainsi que les hymnes liturgiques ont pu influencer la création artistique en Occident et à Byzance, depuis l'époque paléochrétienne.

Deux exemples byzantins ont soulevé notre problématique. Dans l'Annonciation de l'église de Péribleptos de Mistra $^{2}$ (2ème moitié du XIVe siècle) de chaque côté de l'arc triomphal de l'abside, dans le fond de la composition, derrière la Vierge, on trouve une petite colonne avec chapiteau corinthien surmontée d'un vase de fleurs au feuillage abondant (Fig. 1). De même, sur une porte d'iconostase du Musée Byzantin ${ }^{3}$ attribuée à l'école crétoise du XVe siècle, dans l'Annonciation, au premier plan, juste à côté du trône de Marie, une colonne est représentée sur laquelle prend place un vase de fleurs dotées d'un riche feuillage (Fig. 2).

Dans ces deux oeuvres, la colonne avec le vase, n'ayant pas un rôle proprement architectural dans la composition, apparaît comme un motif étrange. Cet élément conduit à se demander s'il représente un motif iconographique de valeur symbolique.

\section{LE VASE DE FLEURS}

Le vase de fleurs est un motif courant dans le sujet de l'Annonciation. Sa signification est bien connue ${ }^{4}$. Il est lié aux textes scripturaires et exégétiques. Le vase est associé au miracle de la manne relaté dans le livre de l'Exode (XVI, 13-34). Suivant les instructions de Moïse,
Aaron a déposé un vase en or rempli de manne dans le tabernacle du témoignage. Ainsi, depuis l'époque paléochrétienne le vase devient le symbole d'une des théophanies prophétiques, qui préfigurent l'Incarnation et par extension Marie et la pureté virginale. Marie est

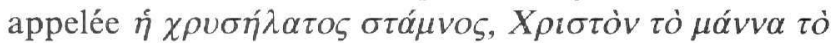
oủpáviov $\varphi \varepsilon ́ \rho o v \sigma \alpha$ (le vase en or qui porte le Christ, la

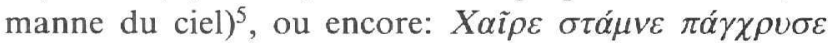

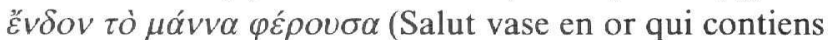
la manne) ${ }^{6}$. Dans un commentaire de Cyrille d'Alexan-

1. Le présent travail avait été communiqué au Congrès d'Art Byzantin et Post-Byzantin de Christianiké Archaeologiké Etaeria, Athènes

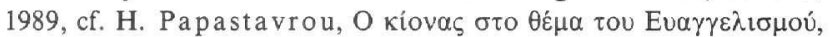
Programme et résumés de communications. Par ailleurs, est faite une brève référence sur le même sujet, dans notre article plus général: Contribution à l'étude des rappors artistiques entre Byzance et Venise. Le thème de l'Annonciation, à paraître in Cahiers Balkaniques (Histoire de l'art), 1990

J'exprime mes remerciements à Mgr. A. Niero, à Venise, pour ses conseils précieux sur le plan théologique. Mes remerciements vont également à Mmes M. Acheimastou-Potamianou, Directrice du Musée Byzantin, pour les facilités de mon travail à ce musée et pour la permission de la reproduction de la photo Fig. 2 provenant de l'archive du Musée Byzantin. D'ailleurs, ma reconnaissance va à Mme C. Baltoyannis dont les suggestions ont toujours été positives à mon travail. Plus particulièrement, sa publication: The Annunciation under a Later Painting on the Royal Door T. 737 in the Byzantine Museum (en grec avec résumé en anglais), AAA XVII (1984), pp. 43-73, a corroboré mon argumentation à une idée qui jusque-là restait difficile à prouver. Enfin j'adresse mes remerciements à Mme D. Mouriki, Professeur en Histoire de l'Art à l'Ecole Polytechnique, pour sa bienveillance de me procurer la photo provenant de l'église de Péribleptos de Mistra (Fig. 1).

2. G. Millet, Monuments byzantins de Mistra, Paris 1910, pl. 116.3.

3. C. Baltoyannis, op.cit. et passim.

4. Sur ce sujet cf. Yrjo Hirn, The Sacred Shrine, Londres 1912, pp. 281-283; D. Robb, The Iconography of the Annunciation in 14th and 15th Centuries, ArtB XVIII (1936), p. 482; D. Mouriki, Ai ßıßдıкаi

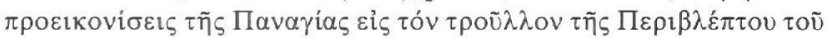

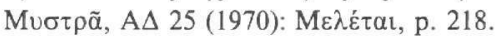

5. PG 97, 865B; une expression pareille se trouve dans un chant à la

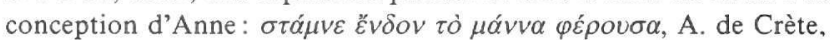
PG 97, 1312A.

6. S. Eustratiades, Theotokarion I, Chennevières-sur Marne, 1931, p. 305 . 


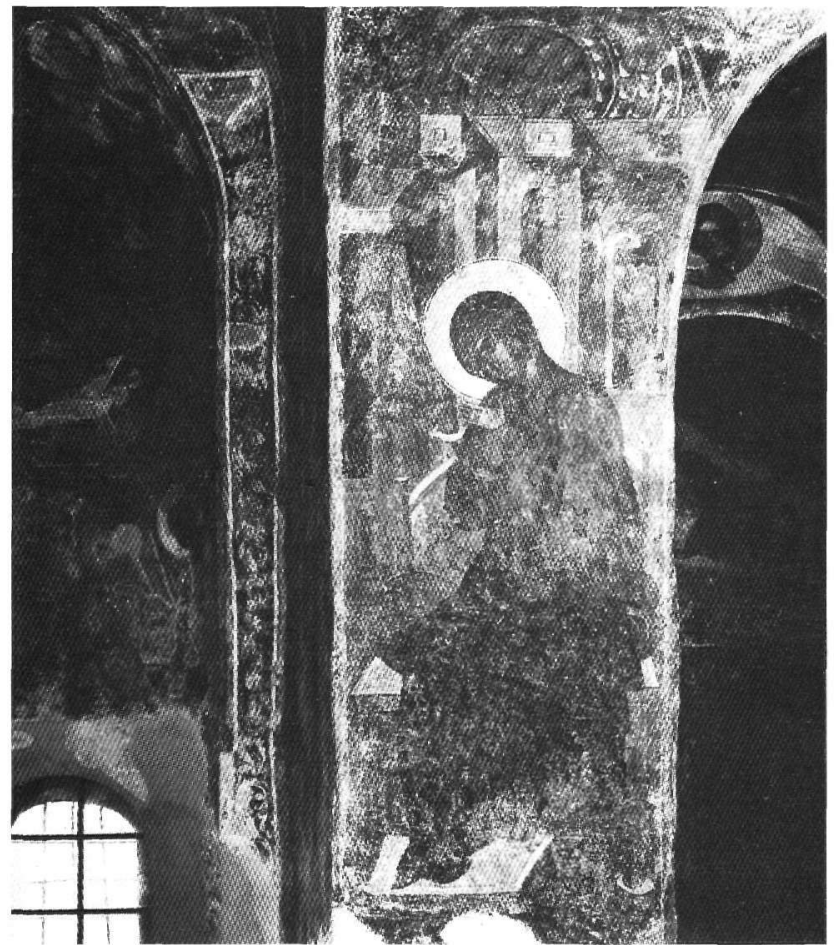

Fig. 1. La Vierge de I'Annonciation, détail. Péribleptos, Mistra.

drie compris dans les Catenae à propos de l'Exode, la manne représente un type du Christ, ce qui indirectement implique que le vase se refère à $\mathrm{Marie}^{7}$. Dans l'iconographie, il se trouve souvent dans les mains de Moïse ou de Aaron, ou alors, dans le contexte de la représentation du tabernacle du témoignage, il se rapproche de la Vierge à l'Enfant ${ }^{8}$.

Dans la scène de l'Annonciation, le vase apparaît régulièrement empli de fleurs et c'est un motif commun aussi bien en Occident qu'à Byzance. Déjà au Xe siècle, il apparaît dans un synaxaire copte (Morgan Library) et dans un manuscrit de Salzburg9. Comme il a été montré ${ }^{10}$, les plantes, l'arbre ou le jardin à côté d'autres images du printemps sont des métaphores, la plupart du temps puisées dans la Bible, qui ont été par excellence utilisées par les auteurs byzantins pour commenter la fête printanière de l'Annonciation. Dans la prophétie d'Isaïe $(11,1)$ p. ex., on mentionne le bâton fleuri. Le Cantique $(2,2)$ cite de même la fleur des champs, le lys ou le jardin clos. Ces métaphores qui représentaient un répertoire dans lequel les loueurs de Marie puisaient constamment ont influencé les imagiers dans leur choix de motifs iconographiques. Ainsi les fleurs dans le vase font allusion à ces images printanières associées à Marie et à la fête même de l'Annonciation.
LA COLONNE DANS LA LITTÉRATURE SCRIPTURAIRE ET PATRISTIQUE

Si dans la scène de l'Annonciation le motif du vase et des fleurs est courant, la colonne représente un élément étrange. Afin de comprendre son rôle nous nous proposons de faire une investigation rapide sur la notion de la colonne dans les textes scripturaires et patristiques et, ensuite, d'évoquer certains exemples en matière artistique qui illustrent sa fonction en tant que symbole.

Dans certains textes de l'Ancien Testament la colonne a une signification cosmologique. Dans le cadre d'une épiphanie divine il y est dit que Dieu fait trembler les fondements de la terre ou du ciel qui originellement ont été créés par Lui-même ${ }^{11}$. Dans ces cas, fondement ou colonne ( $\sigma \tau \tilde{v} \lambda \circ \varsigma, \theta \varepsilon \mu \varepsilon \dot{\varepsilon} \iota \mathrm{tov}, \sigma \tau \varepsilon \rho \varepsilon ́ \omega \mu \alpha)$ sont des termes synonymes ${ }^{12}$. L'image qui est sous-entendue est celle d'une maison construite par Dieu, image qui à l'époque paléochrétienne a été appliquée en Orient à l'église. Par ailleurs, la même idée se retrouve lorsqu'il s'agit du rôle de la sagesse en tant que médiatrice de la création qui "place son trône sur une colonne de nuage"13. Dans un autre passage il est écrit de même que la sagesse a construit sa maison sur sept colonnes ${ }^{14}$. Egalement, la colonne de nuage ou de feu est ou bien un signe de la présence de Dieu qui montre le chemin à suivre ${ }^{15}$ ou alors, une désignation de Sa présence dans le contexte des idées relatives au culte de Dieu et au tabernacle ${ }^{16}$. Chez Jérémie la "columna ferrea", d'après l'exégèse chrétienne ${ }^{17}$, se refère à l'Eglise.

Dans la littérature patristique, pour sa part, l'élément de la colonne est chargé de sens et de rapprochements, parmi lesquels quelques-uns nous aideront à appuyer nos arguments. Les textes exégétiques du moyen âge, bien sûr, utilisent abondamment des citations bibliques et font un grand usage de la métaphore de la colonne. Les Pères de l'Eglise orientale par la colonne symbolisent tantôt le Christ, comme Origène ${ }^{18}$, tantôt l'Eglise ${ }^{19}$ ou l'Evangile ${ }^{20}$, tantôt les apôtres ${ }^{21}$, les saints ${ }^{22}$ ou les archevêques ${ }^{23}$. D'autre part, parmi les théologiens latins, Saint Grégoire le Grand ${ }^{24}$ écrit que les colonnes du ciel sont les grands prédicateurs de l'Eglise ou des églises-lieux de culte chrétiens. Pour Raban Maur ${ }^{25}$ les colonnes sont les apôtres, les prédicateurs, mais aussi le progrès de la foi et la conduite de la vie suivant l'esprit chrétien. Il affirme également que les colonnes signifient les actions du Christ ${ }^{26}$, ses vertus ${ }^{27}$, ou bien encore, le Christ Lui-même ${ }^{28}$. Alcuin compare les colonnes aux sept dons du Saint-Esprit ${ }^{29}$. Comme la Sagesse a construit sa maison sur sept colonnes (Prov. 9, 1), le Christ a renforcé sa maison, l'Eglise, avec les sept dons du Saint-Esprit. Alain de Lille ${ }^{30}$, d'autre part, compare la 


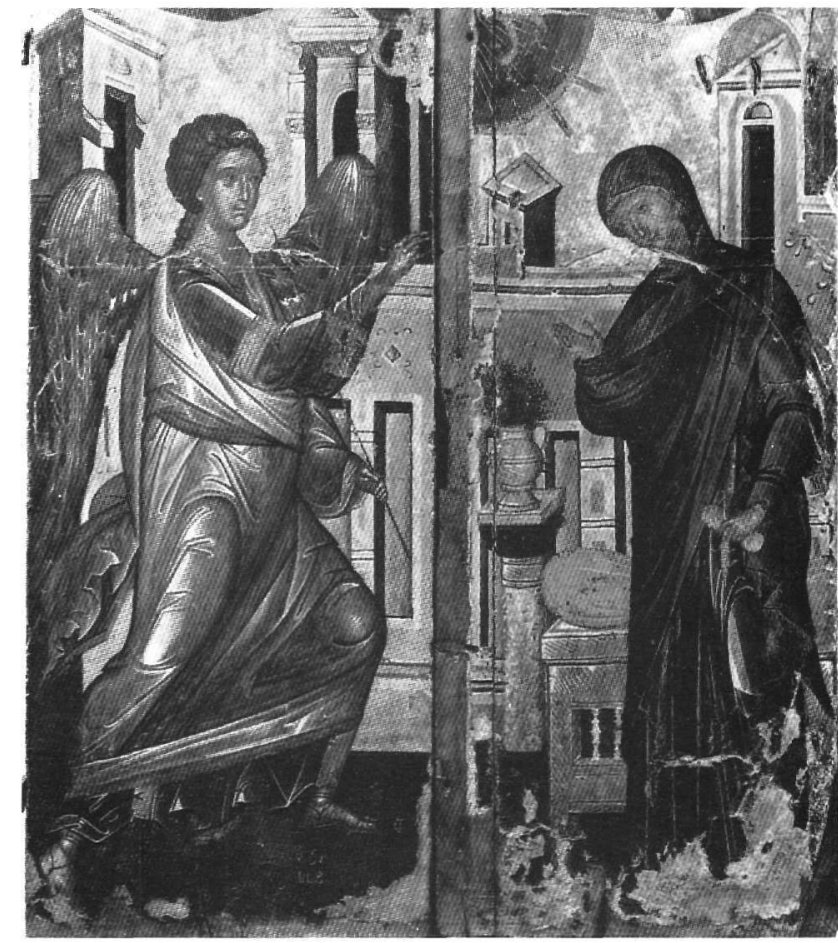

Fig. 2. L'Annonciation sur la porte d'iconostase no. T. $737 \mathrm{du}$ Musée Byzantin.

Sagesse à la sainte Vierge qui possédait les quatre vertus cardinales de sagesse, de tempérance, de force et de justice et les trois vertus divines de foi, d'espoir et d'amour. La description de l'abbé Suger ${ }^{31}$ de l'église de Saint-Denis met bien en évidence que les colonnes représentent les douze apôtres et les prophètes, alors que le Christ Lui-même est la pierre angulaire, métaphore qui revient maintes fois dans les textes patristiques. L'abbé Gibert ${ }^{32}$ de Sainte-Marie à Novigento (1104) écrit: "Christ qui était Lui-même une colonne crée les prototypes de la rectitude et de la force". Saint Bruno ${ }^{33}$ dit que la nature humaine du Christ est appelée colonne, car le support de notre foi est fort comme une colonne. Enfin, pour le

7. Ce commentaire a été compris parmi les Catenae à l'Exode, cf. $\mathrm{N}$.

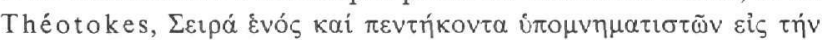

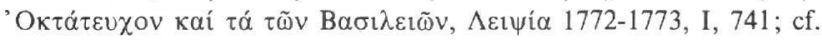
Mouriki, op.cit., p. 220 , note 10 .

8. Mouriki, op.cit. et passim.

9. Robb, op.cit., p. 482.

10. H. Maguire, Art and Eloquence in Byzantium, Princeton 1981, pp. 42 sq.

11. Grande Lessico del Nuovo Testamento, XII, 1393.

12. I. Tim. 3,$15 ;$ Job 9,$6 ; 38,6 ; 26,11$.

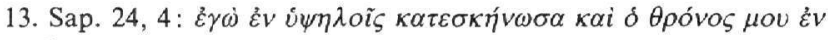
$\sigma \tau \hat{\lambda} \omega \nu \varepsilon \varphi \dot{\varepsilon} \lambda \eta \zeta$

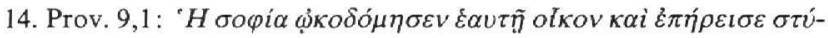

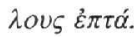

15. Ex. 14 , 19. $24 ; 19.9$; Num. 14, 14; Neem. 9, 12.19; Sap. 18, 3.

16. Num. 12,5 ; Deut. 31,15 ; ps. 98,7 .

17. Jer. I, 18: Ego quippe dede te hodie in civitam munitam et in columnam ferream et in murum aureum, super omnem terram.

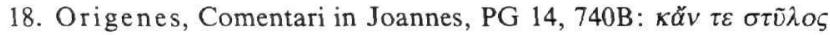

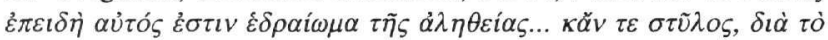
$\varphi \omega \tau \varepsilon ı v o \dot{v} \tau o \tilde{v} \pi v \rho o ́ \varsigma$; Epiphanius Constantensis, Adversus haereses, PG 42, 256D.

19. Theodorus Mopsuestenus, Epistolae Beati Pauli, I ad Timo-

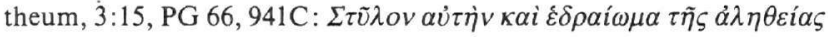

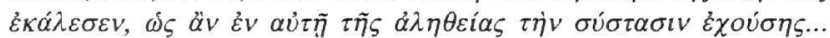

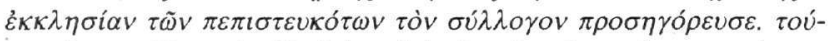

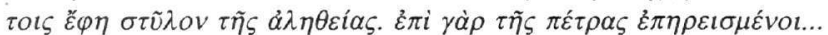

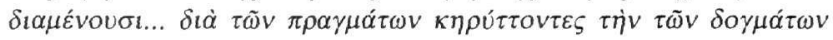
$\dot{\alpha} \lambda \eta \dot{\theta} \theta \varepsilon \iota \alpha v$

20. Irenaeos Lugdunensis, PG 7, 885A.

21. Cyrillus Alexandrinus, De adoratione in spiritu et veritate, PG 68, 133 .

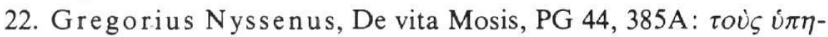

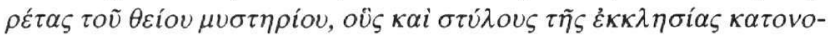

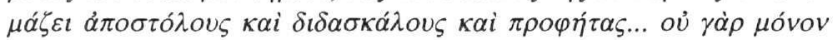

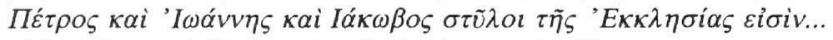

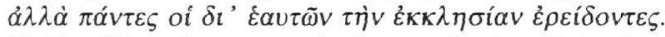

23. Basilius Caesarensis Cappadociae, Epistulae, PG 32, 908C. 24. Saint Grégoire le Grand, Moralium libri, Liber XVII, caput XXIV beati Job; ph. LXXVI: 30 .

25. Rabanus Maurus, Allegoriae in sacram scripturam, PL CXII: 899-900. Per 'colomnas' apostoli, ut in Paulo: 'Jacobus et Cephas et Joannes, qui videbantur columnae esse', quod illi et caeteri apostoli Ecclesian sustentant. Per 'columnam praedicatores, ut in Exodo: 'Facies columnas viginti, cum totidem basicus', id est, constitues praedicatores sanctos, qui utrumque testamentum intelligentes prophetarum doctrinis initiantur. Per 'columnas' Scripturae sacrae, ut in Cantico: 'Columnas ejus fecit argenteas', quod scripturas in sancta Ecclesia fecit noster pacificus Christus intellectum daturas. Per 'columnam' rectituto fidei et strenuitas vitae, ut in libris Regum: 'Statuit duas columnas in introitu templi, quod celestis patriae introitum introire nequimus, nisi et recte credamus, et sancte vivamus.

26. I dem, op.cit.: Per columnas actiones Christi ut in Cantico: 'Crura ejus sicut columnae marmoreae' quod actiones Christi et rectae sunt et fortes.

27. I d e m, op.cit., PL CXII: 900: Per columnas internae virtutes mentis ut in Psalmis: 'Ego confirmavi columnas ejus (Liber Psalmorum LXXIX, 4)' Id est ego roboravi internas virtutes ejus.

28. Idem, op.cit., PL XCII : 899: Columna est divinitas Christi... Columna humanitas Christi...

29. Alcuin, Epistole, epistola CCIII, PL C: 477.

30. PL CCX: 262.

31. Suger, Libellus de consecratione ecclesiae sancti Dionysii et traslatione corporum sancti Dionysii ac sociorum ejus; cité par E. Panowski, Abbot Suger on the Abbey Church of Saint Denis and its Art and Treasures, Princeton, New Jersey 1946, pp. 104-105.

32. Gibertus, Gesta Dei per francos, Liber VIII, caput V, PL CLVI: 810: Christus ipse columna rectitudinis ac fortitudinis inspiravit exempla.

33. Saint-Bruno, Expositio in Psalmos, psalmus XCVIII, PL CLII: 1157: Convenienter etiam humanitas columna dicitur eo quod admodum columnae firmissimum sit fidei nostrae sustentaculum. 


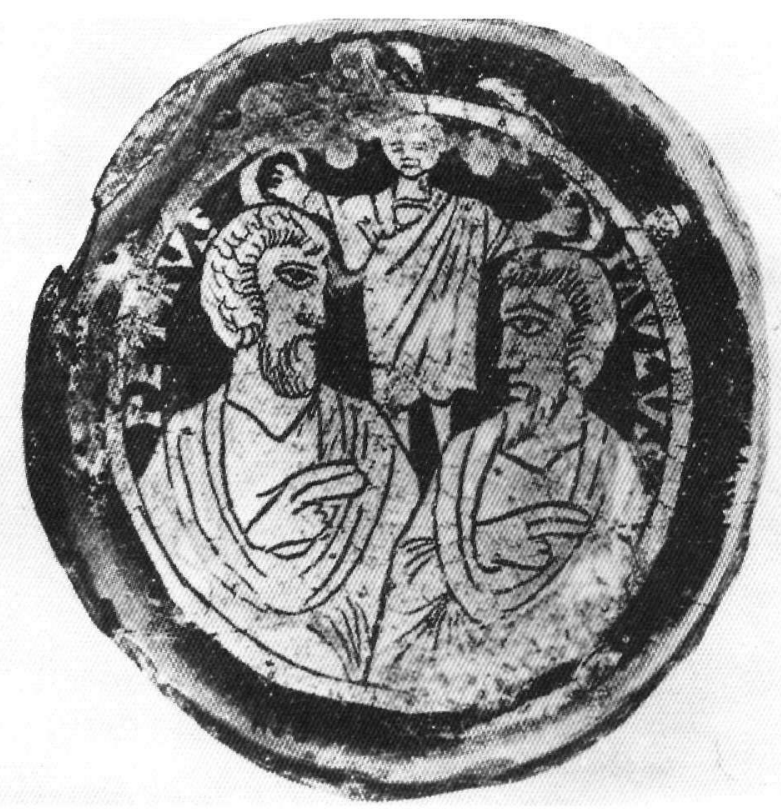

Fig. 3. Composition sur verre. Musée Vatican.

moine benedictin Candidus ${ }^{34}$, à propos de Eigil qui a construit l'église de Fulda, le Christ est la fondation et la colonne de cette église et celle-ci restera immobile pour toujours grâce au pouvoir de sa majesté perpetuelle. Ces textes ont sans doute servi à l'inspiration artistique dès les premiers siècles chrétiens, puisque la première référence à la colonne est faite dès le Nouveau Testament (I Tim. 3: 15; I Cor. III, 9-17; II Cor. V 1-5; Eph. II, 20-21; Gal. II, 9). Ainsi, comme il sera évoqué à la suite de cette étude, nombreux exemples paléochrétiens et du moyen âge témoignent d'un rapport étroit entre le motif de la colonne dans l'art et dans la littérature.

\section{LA COLONNE DANS L'ART PALÉOCHRÉTIEN ET DU MOYEN AGE OCCIDENTAL}

Une série de représentations sur verre d'époque pale'ochrétienne faisant partie de la collection de la Bibliothèque Vaticane représente le Christ imberbe en tunique et pallium avec un couple marié. Au dessus de la tête de chaque époux le Seigneur tient une couronne ${ }^{35}$ (Figs. 3, 4) Sur d'autres exemples, le Christ est représenté entre Pierre et Paul en tenant une couronne au dessus de la tête de chacun d'eux ${ }^{36}$. Dans des compositions similaires ${ }^{37}$ à la place de l'image du Christ, on voit une colonne surmontée par Son monogramme $\mathbb{R}$. Ainsi, sur un autre verre deux apôtres, qu'une inscription désigne:

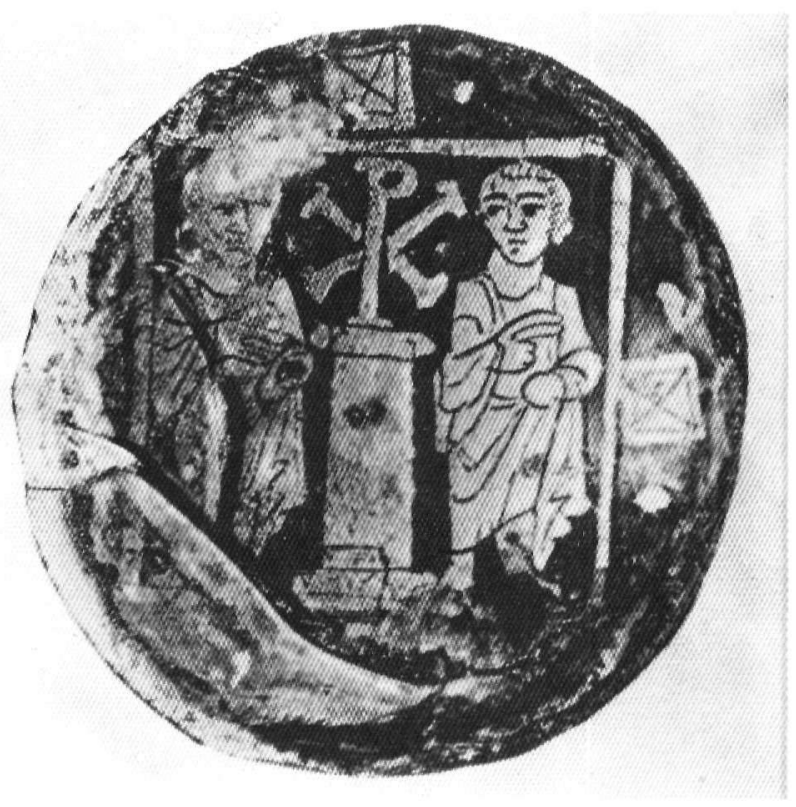

Fig. 4. Composition sur verre. Musée Vatican.

PETRUS PAULUS, sont représentés frontalement; une colonne est placée entre eux deux. Au dessus d'elle est placé un médaillon dans lequel est inscrit le monogramme du Christ ${ }^{38}$. Dans ces exemples il est clair que l'image du Christ même est remplacée par la présence de la colonne, ce qui montre la valeur symbolique dont ce motif est investi.

Ce fait est explicite dans une autre oeuvre paléochrétienne. Il s'agit d'un sarcophage provenant de SaintAmbroise de $\operatorname{Milan}^{39}$ (Fig. 5). Dans la scène de la face postérieure, le Christ apparaît entouré de ses apôtres. A ses pieds se dresse l'Agneau entre les deux defunts prosternés. Le groupe central du Christ et des Princes des apôtres semble encadré à la partie supérieure, par les éléments d'une architecture qui rappelle un portique. Tandis que la tête du Christ se détache au centre de l'architrave incurvée et devant un chapiteau plus grand que les autres, celles des deux apôtres sont situées immédiatement au-dessous des chapiteaux. Assis très exactement devant les colonnes qu'ils masquent, les apôtres semblent se substituer à celles-ci en leur empruntant leur symbolisme. D'après les textes scripturaires et exégétiques $^{40}$, la composition de cette image symbolise peut-être l'édification du temple de l'Eglise par l'enseignement du Christ, qui en est sa pierre angulaire, et par la prédication des apôtres, qui en sont ses colonnes.

Par ailleurs, dans une enluminure du IXe siècle ${ }^{41}$, Paris. gr. 510, fol. 452 (Fig. 6), toute une page est occupée par 


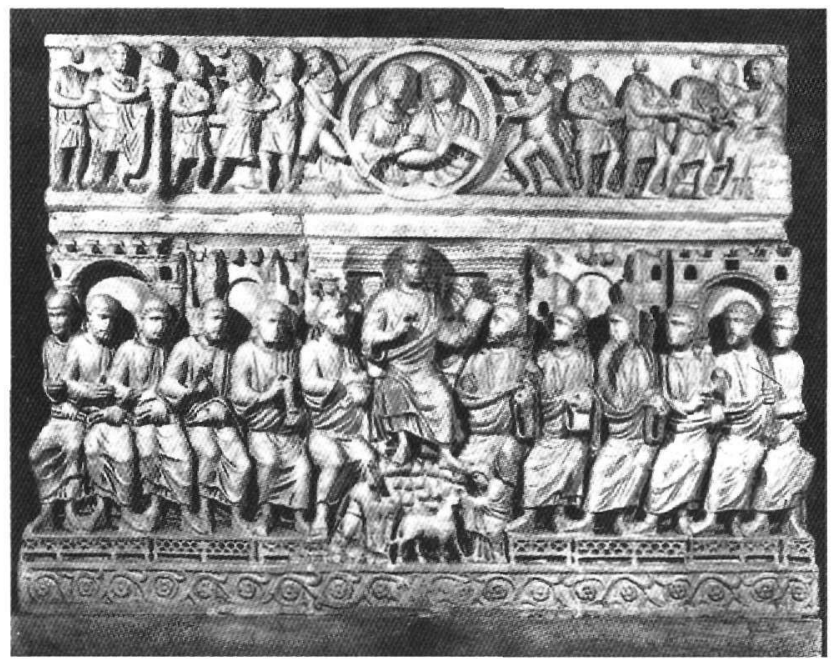

Fig. 5. Face postérieure de sarcophage. Saint-Ambroise, Milan.

Fig. 6. Cod. Paris. gr. 510, f. 452. Scènes de la vie de saint Grégoire.

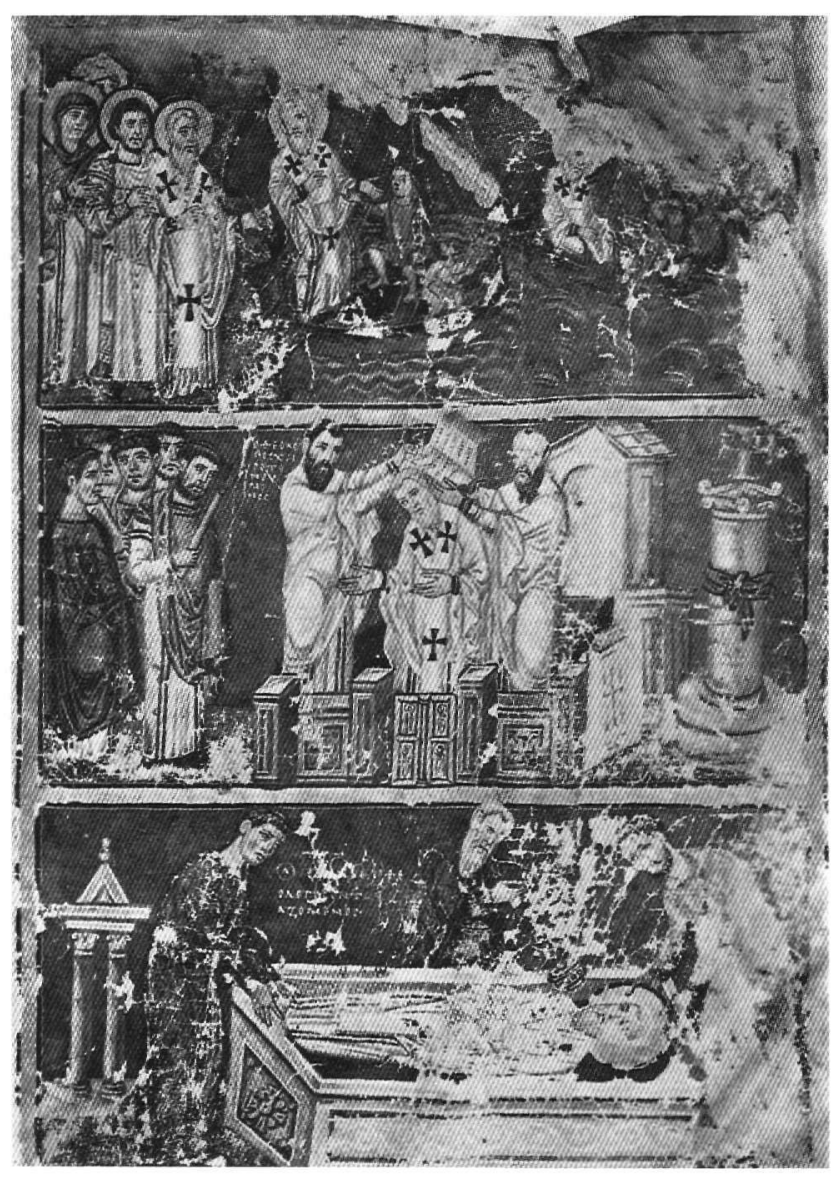

trois scènes de la vie de saint Grégoire. Dans la scène médiane, il s'agit de la consécration du hiérarche. Saint Grégoire est placé au centre, debout et en orant. De chaque côté, deux personnages tiennent l'Evangile au dessus de sa tête. A l'arrière, s'élève une construction. A gauche, un groupe de personnages témoignent de l'événement et à droite une colonne avec base et chapiteau surmontée d'une croix limite la composition. La présence de la colonne est ici plus qu'un élément décoratif. Une des interprétations possibles de ce motif serait qu'il représente la qualité de saint Grégoire en tant que colonne de l'orthodoxie. Pourtant, une autre explication semble plus probable. La colonne illustre peut-être l'idée de l'Eglise. Car, dans le même manuscrit (Fig. 7) une autre scène qui représente également la consécration du hiérarche se déroule devant un ciborium.

Dans son étude sur la signification des éléments de l'architecture religieuse romane, $\mathrm{F}$. Carlsson ${ }^{42}$ suggère que certains éléments architecturaux non fonctionnels étaient utilisés surtout pour des raisons symboliques. Partant de l'observation que dans certaines églises de la province romaine des premiers siècles chrétiens des colonnes se trouvent placées sans raison fonctionnelle dans la nef ${ }^{43}$ ou dans l'abside ${ }^{44}$, alors que le même phé-

34. Candidus de Fulda, Sancti Eigilii vita, PL CV: 397.

35. C. R. Morey, The Gold-glass Collection of the Vatican Library, Vatican 1959 (MCMLIX), Nos. 109, 240, 397.

36. Idem, op.cit., nos. $241,286,314$.

37. Id em, op.cit., nos. $76,79,88,98$

38. Idem, op.cit., no. 455 .

39. M. L. Thérel, Les symboles de l'"écclesia", dans la création iconographique de l'art chrétien du IIIe au VIe siècle, préface de J. Danielou, Rome 1973, p. 111.

40. Dans la littérature chrétienne des premiers siècles l'Eglise est présentée sous l'image d'un édifice dont le Christ est la pierre angulaire, cf. : I Cor., III 9-17; 2 Cor., V 1-5; Eph., II 20-21; Saint-A mbroise, Comment. In epist. ad Corinth. Prim., PL XVII, PG XXII: 1070, 1082C. De même les apôtres sont comparés à des colonnes: Gal. II, 9; Saint-Augustin, PL XXXIII : 853; XXXVI : 950; XXXVII : 1831; Saint-Ambroise, PL XV: 1264; Saint-Paulin d'Aquilée, PL XCIX: 183.

41. S. der Nersessian, The Illustrations of the Homilies of Gregory of Nazianzus, Paris. gr. 510. A Study of the Connections between Text and Images, DOP 16 (1962), fig. 10.

42. F. Carlss on, The Iconology of the Tectonics in Romanesque Art, Hasslehorl 1976.

43. Il s'agit de la basilique de Tebessa en Tunisie, cf. A. Grabar, The Beginnings of Christian Art (The Arts of Mankind), Londres 1967, figs. 198, 199; de Deir-el-Akhmar et Deir-el-Abiad en Egypte (c. 400), cf. idem, Byzantium (The Arts of Mankind), Londres 1967, figs. 34, 35 pour le premier monument et fig. 36 pour le deuxième.

44. Il s'agit des églises syriennes de Qalaat Seman: cf. A. G raba r, op. cit., fig. 53; et Qalbloze: ide m, op.cit., figs. 44, 45. Ces églises ont une série de colonnes sans fonction architecturale dans l'abside. 
nomène est repris dans les églises carolingiennes ou mosarabes des IXe, Xe, et XIe siècles ${ }^{45}$, il constate que les éléments non fonctionnels sont nombreux dans l'architecture romane ${ }^{46}$. Pour ne citer que quelques exemples, douze colonnes antiques en marbre ont été placées dans le choeur de la Cathédrale de Magdeburg (1209) que Carlsson interprète comme une représentation des douze apôtres ${ }^{47}$, comme autrefois à Jérusalem, au SaintSepulcre, 12 colonnes, les 12 apôtres gardaient la tombe du Seigneur ${ }^{48}$. Pour la même raison, les six colonnes à l'extérieur de l'abside de l'église de Perse s'appuient sur six pilliers (Fig. 8). Le motif frappant de la niche absidiale avec une seule colonne dans quatre églises scandinaves a été expliqué en tant que symbole du Christ ${ }^{50}$. Egalement, ce même motif est placé au milieu de plusieurs tympans comme p.ex. à l'église diocésaine de Gernrode où il se trouve dans le cloître ${ }^{51}$ ou dans l'église paroissale de Griesheim, sur le portail sud ${ }^{52}$. Le symbolisme du Christ en tant que pierre angulaire de l'Eglise est evident dans les grands portails de certaines cathédrales en France comme celle d'Oloron dans les Pyrénées-Atlantiques (Fig. 9). Le pilier central, qui est de style dorien-toscan, diffère de ceux des côtés. Il est soutenu par deux atlantes et sur le côté frontal de son chapiteau apparaît une croix. Ce pilier est placé sur un axe, au prolongement duquel est disposé, chacun par dessus l'autre un certain nombre de symboles christologiques: le monogramme du Christ qui sert de base à la scène de la Descente de la croix, la tête d'un lion et plus haut, l'Agneau.

Un autre exemple roman qui met bien en évidence la valeur symbolique de la colonne provient de l'ambon d'une église d'Italie du Sud, à Trasacco ${ }^{53}$ (Fig. 10). Au centre d'une composition, l'agneau et une colonnette occupe la place d'un Christ en majesté. Ces motifs sont entourés par les symboles des quatre évangélistes et par des éléments cosmiques, le soleil, la lune, et des planètes. Dans ce cas, une représentation de la colonne de la passion est difficilement admise, car, du bas de ce motif, aussi bien que par la base de son chapiteau poussent de longs rinceaux qui contiennent les planètes comme s'il s'agissait de fleurs. Ainsi, en combinaison avec l'agneau, la colonne devient ici le symbole du Christ créateur en tant que pierre angulaire de l'univers ${ }^{54}$. Par ailleurs, dans d'autres exemples la colonnette est souvent flanquée par quatre rosettes, autres symboles des évangélistes $^{55}$.

Les exemples innombrables de l'art occidental montrent clairement combien la colonne y devient un motif de valeur symbolique importante pendant le moyen âge. Son caractère vivant se révèle également dans un autre élément significatif pour notre étude. Au XIIe siècle

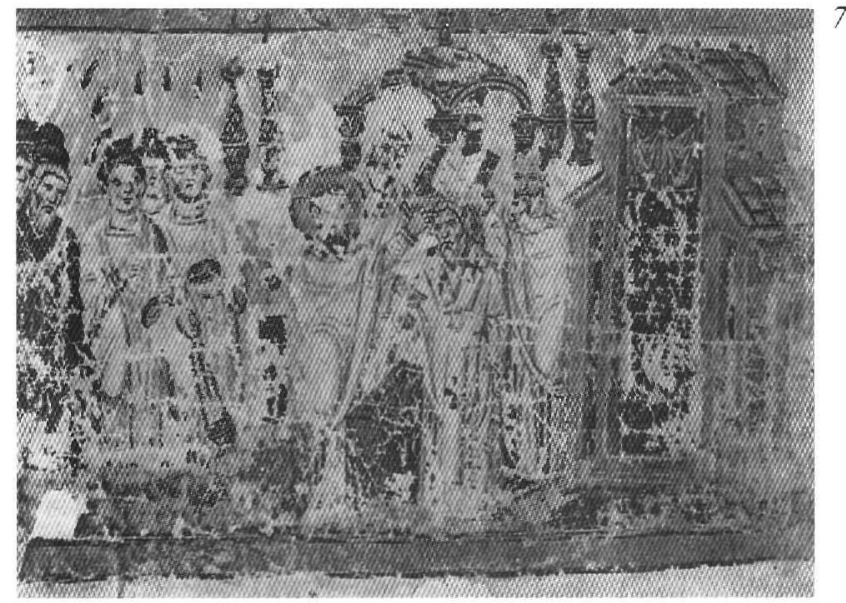

Fig. 7. Cod. Paris. gr. 510, f. 67v; détail avec la scène de la consécration de saint Grégoire.

Fig. 8. Eglise de Perse, vue de la partie Est.

Fig. 9. Cathédrale d'Oloron, portail ouest.

Fig. 10. Ambon de Trasacco.

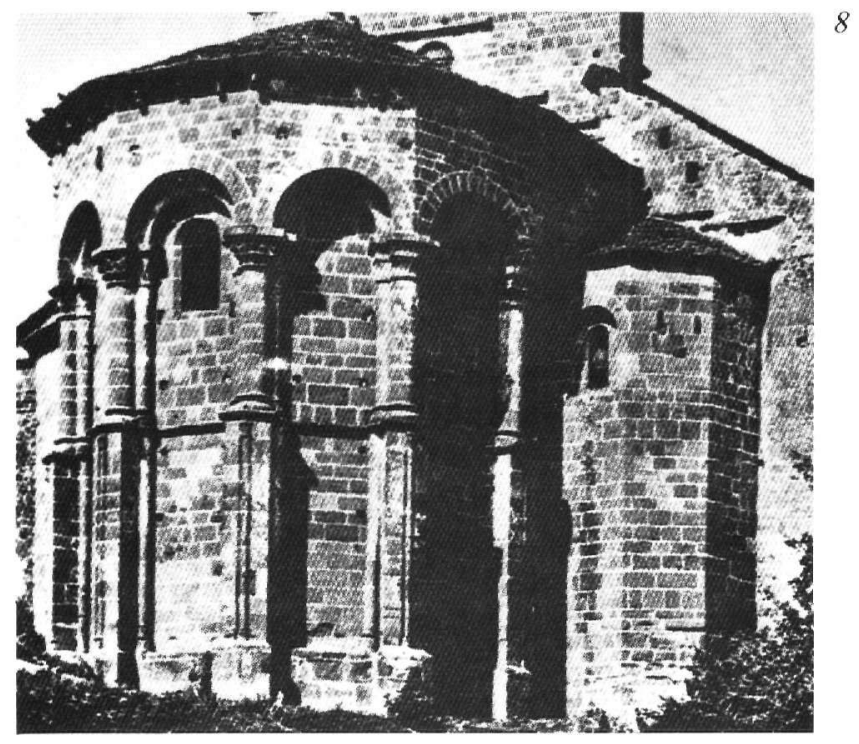

saint Bonaventure ${ }^{56}$ raconte que, pendant la naissance du Christ, Marie s'est appuyée sur une colonne et a accouché sans douleurs. Ainsi, par une autre voie indirecte, la colonne se lie à l'Incarnation et, par extension, à la Vierge. Elle devient symbole "virginitatis in partu". Cette idée est reprise en matière artistique. Les peintres flamands, on le sait, font un usage abondant de sym- 
boles. Pour ne citer que quelques exemples, dans le "retable de Bladelin" (Kaiser Friedrich Museum, Berlin) de R. van der Weyden (Fig. 11), la scène de la Nativité du Christ est placée dans une humble construction très déteriorée. Au premier plan est affichée une colonne en marbre avec base et chapiteau. Une mise en scène semblable se trouve dans l'oeuvre de Hugo van der Goes dans le retable Portarin (Uffizi, Florence) (Fig. 12), ainsi que dans d'autres exemples ${ }^{57}$.

Nous arrivons donc à une conclusion. Jusqu'à présent, les exemples de l'art paléochrétien et occidental du moyen âge ont montré que le motif de la colonne peut être un symbole de l'Eglise ou du Christ. Bien entendu, également, il se lie au thème de l'Incarnation et, par extension, à la Vierge.

45. P. ex. Saint-Germigny-des-Prez: J. Huber, J. Porcher, W. F. Volbach, Carolingian Art (The Arts of Mankind), Londres 1970 , figs. 42, 43; San-Pedro-della-Nave, cf. J. Fontaine, L'art préroman hispanique (La pierre qui vire), 1973, fig. 68; San-Pere-de-Roda, cf. Carlsson, op.cit., fig. 2.

46. P. ex. dans la cathédrale de Hildesheim ou de Dolby en Suède, une colonne était placée dans chaque niche des pilliers occidentaux de la nef. Ces colonnes allégeaient les pilliers dont elles faisaient partie, mais, sûrement, en même temps, elles communiquaient un message; cf. Carlsson, op.cit., fig. 3.

47. Carlsson, op.cit., fig. 5 .

48. G. Bandmann, Zur Bedeutung der romanischen Apsis, WallradRichards-Jahrbuch XV (1955), cité par Carlsson, op.cit., p. 54.

49. Carlsson, op.cit., fig. 6.

50. Il s'agit des églises de Fulltofta, Bosjo, Norre Jernlose et Rutsker; cf. B. Thyren, Den kolonettdelade nischen a Bosjo Klosterkyrkas absid samt dess mostvarigheter i Fulltofta, Norre Jernlose och Rutsker (dissertation non publiée, Lund 1968; cité par Carlsson, op.cit., p. 54.

51. W. Blankenburg, Heilige und demonische Tiere, Leipzig 1943, fig. 68 .

52. R. Beuerreiss, Das Lebenszeichen, Veröffentlichungen der Bayerischen Benediktinerakademie, I (1961), fig. 28

53. R. Reuterswärd, The Forgotten Symbols of God. Five Essays Reprinted from Konsthistorisk Tidskrift (Stockholm Studies in History of Art, 35), Uppsala 1986, p. 110.

54. Cette représentation rappelle la composition avec le motif central d'une roue par où poussent des rinceaux qui contiennent des planètes, alors que les "zodias" des évangélistes prennent place autour de ce schéma central, cf. Reuterswärd, op.cit. et passim.

55. Reuterswärd, op.cit., passim.

56. Cf. G. Schiller, Ikonographie der christlichen Kunst, Guterslocher, 1975, 1, pp. 86, 87

57. E. Panowsky, Early Netherlandish Painting. Its Origins and Character, Cambridge, Mass. 1964, 2, pls. 152, 153 etc.
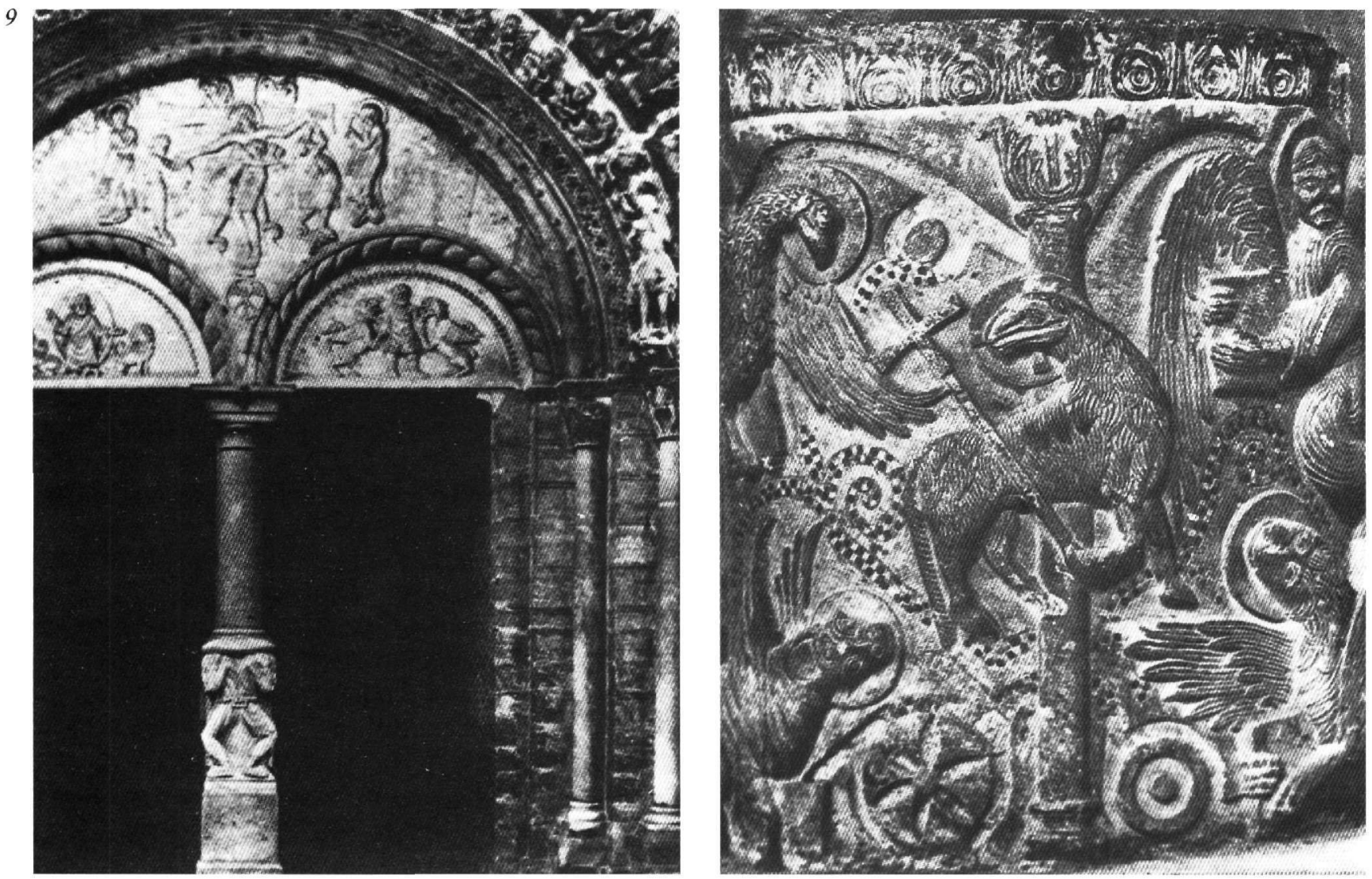

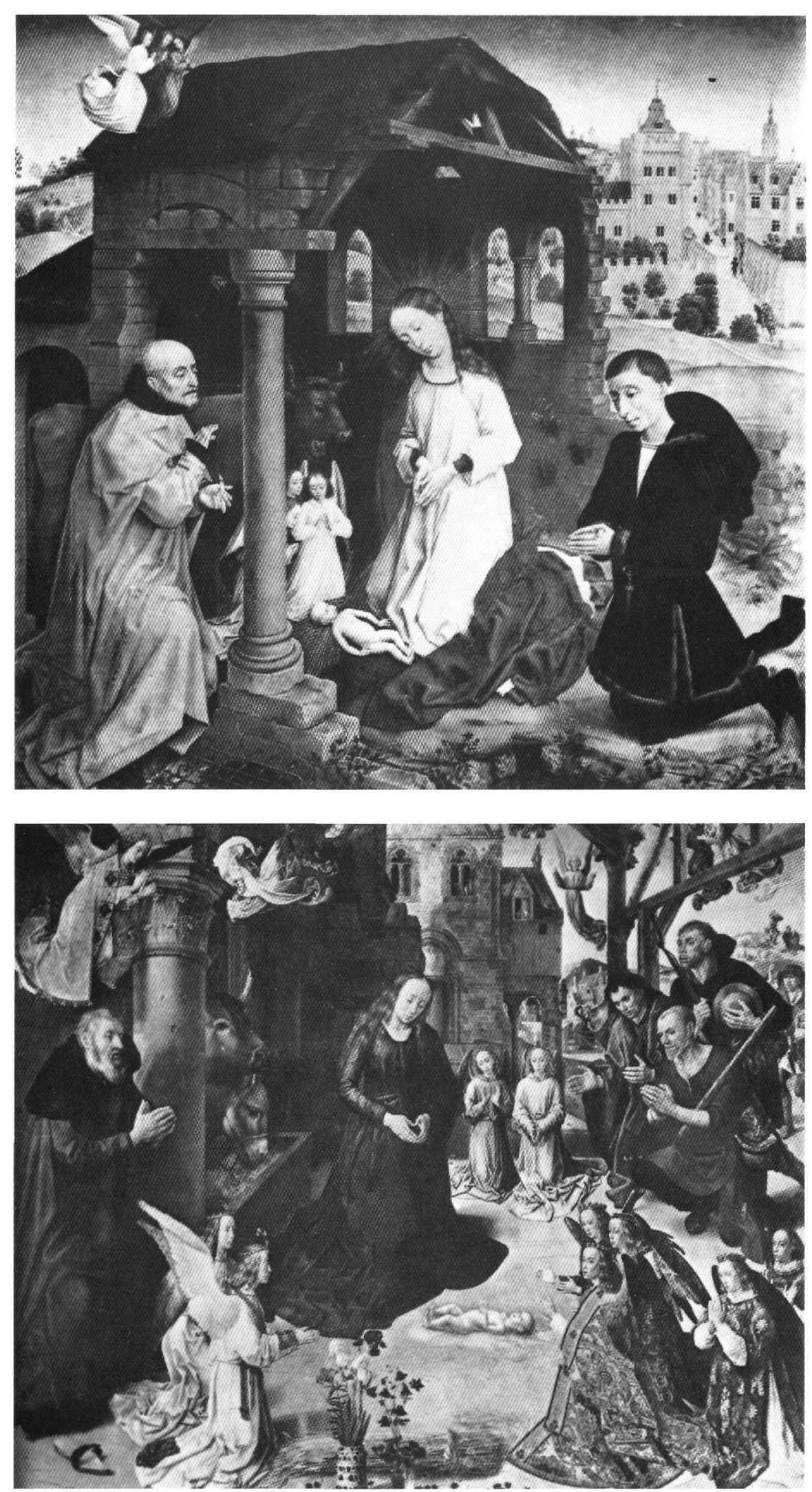

Fig. 11. Le retable "Bladelin" de Roger van der Weyden; détail avec la Nativité. Kaiser Friedrich Museum, Berlin.

Fig. 12. Retable Portarin de Hugo van der Goes; détail avec la Nativité. Uffizi, Florence. 
LE RÔLE DE LA COLONNE DANS L'ANNONCIATION EN OCCIDENT

Dans la tradition picturale de l'Annonciation du moyen âge occidental, la colonne se place ou bien à l'angle de l'édicule de Marie, ou alors, faisant partie d'une arcature, elle crée des lieux figuratifs diversifiés pour chacun des protagonistes. Dans certaines Annonciations occidentales du XVe siècle, la colonne a un emplacement remarquable dans la disposition de la composition. Quelquefois, elle occuppe un poste clé dans l'agencement de la composition, comme p. ex. chez A. Lorenzet$\mathrm{ti}^{58}$, qui l'utilise pour occulter le point de fuite de son pavement, ou chez P. della Francesca ${ }^{59}$ et chez Antonello da Messina ${ }^{60}$ (Fig. 13) la colonne devenant le centre de gravité de l'action. Dans d'autres cas, au contraire, sa présence n'a pas de justification proprement architecturale devenant ainsi un élément absurde. C'est le cas d'un panneau de l'Annonciation de Masolino (Fig. 14), conservé à Washington. Contrairement à ce qu'on a vu antérieurement, la colonne ici met en valeur le point de fuite de la perspective, de sorte que D. Arasse ${ }^{61}$ a suggéré qu'il s'agit là d'un motif symbolique. Du reste, d'autres exemples comportent cet élément de façon plus ou moins suggestive. Dans une enluminure ${ }^{62}$ de la fin du XVe siècle représentant le thème de l'Annonciation, sur le pupitre de Marie s'élève une colonne avec chapiteau surmontée d'une sculpture (Fig. 15). Par ailleurs, dans l'Annonciation attribuée à Hubert van Eyck (?), deux colonnes sont placées sur la façade de la maison de Marie, symboles de l'Ancien et du Nouveau Testament ${ }^{63}$. Pourtant, le plus ancien exemple de l'Annonciation que nous connaissons, où l'on peut considérer que la présence du motif de la colonne transmet probablement un message, provient de l'enluminure d'un évangéliaire marcien (cod. lat. I, 100) de la première moitié de XIVe siècle (Fig. 16). Sur l'axe médian de la composition on voit superposés un certain nombre de motifs: un socle d'où de l'eau jaillit, une colonne et un jardin de palmiers. Comme nous l'avons montré dans une autre étude ${ }^{64}$, le fait que la colonne pourrait être une métaphore est corroboré par l'association de celle-ci à d'autres éléments iconographiques, dont la valeur symbolique est plus claire: le jardin clos avec la plante paradisiaque, le palmier, et la source qui sont des métaphores connues et associées à Marie.

Il semble qu'en effet, dans l'art occidental, le symbole vivant de la colonne rattachée à l'image du Christ ou, en tous cas, à l'ensemble du thème de l'Incarnation, tout comme les homélies théologiques ou des textes plus populaires comme celui de Bonaventure, ont également influencé la scène de l'Annonciation. Des textes litur- giques latins louent la Vierge pour son accouchement sans douleurs (dont le symbole est devenu, on l'a vu, la colonne), pour sa sainteté et son rôle en tant que mère et fondement, de Dieu: "pulchrae sunt genae tuae sicut turturis... sancta Dei genitrix fundamenta ejus"65. Ainsi, une nouvelle nuance s'attache au motif iconographique de la colonne, qui s'associe à Marie, dans la scène de l'Annonciation occidentale, à la fin du moyen âge, et qui accentue son rôle en tant qu'instrument de l'Incarnation. Par ailleurs, dans la même scène en Occident, on met l'accent plus spécialement sur l'idée de l'Incarnation que sur le message angélique. Dans maints exemples, l'Enfant (souvent avec la croix) descend du ciel et se dirige vers Marie ${ }^{66}$. Une oeuvre de Nicola da Guardagrele (entre 1433 et 1448) (Fig. 17) et d'une "Liber viaticus" (vers 1355-1360) ${ }^{67}$, où l'Ange lui-même s'approche de Marie et lui confie l'Enfant, constituent des exemples particulièrement intéressants. Dans un "Liber dipictus" (vers 1358) entre deux scènes, l'Annonciation et la Nativité, on voit assez curieusement Jésus Christ bénissant Marie: l'Incarnation est comprise ici comme le choix de l'Epouse du Logos; la phrase de l'hymne "Ave Sponsa insposata" constitue la clé qui permet de comprendre ce symbole ${ }^{68}$.

Nous retenons donc, que dans les Annonciations occidentales que nous avons mentionnées, la colonne, outre

58. E. Panowsky, Perspective comme forme symbolique, Paris 1978 , p. 124 , pl. 22.

59. M. Baxandall, Painting and Experience in 15th Century in Italy, Oxford 1980, fig. 1

60. Robb, op.cit. (n. 4), fig. 41.

61. D. Arasse, Espace pictural et image religieuse; le point de vue de Masolino sur la perspective, tiré à part, p. 143, fig. 7a et 7b. L'auteur explique que certaines particularités de Masolino dans sa façon de rendre la perspective étaient dues à une volonté certaine du peintre de faire surgir dans l'espace mesurable des figures ce qui lui échappe, c'est à dire, le spirituel non mesurable. Le peintre était, sûrement, proche des sermons et des textes exégétiques, dont l'influence porte dans son oeuvre. Car, il est attesté qu'il se liait au cardinal Branda Castiglione, son commanditaire et conseiller "iconographique". Dans la scène de l'Annonciation, suggère l'auteur, la colonne symbolise le Christ.

62. Livres d'heures, Cod. Guelf. 56.8 Augusteus 40, fol. 29r; cf. W. Milde, Mittelalterliche Handschriften der Herzog August Bibliothek, Frankfurt am Main 1972, p. 228.

63. Cf. LChrI, Säule, col. 55. Le symbolisme des deux Testaments par deux colonnes peut se constater également en architecture comme p. ex. sur la façade ouest de Saint-Michel à Pavia ou sur la façade ouest de la Cathédrale de Piacenza; cf. Carlsson, op.cit., p. 54.

64. H. Papastavrou, Rapports artistiques (n. 1), et passim.

65. Breviarium Romanum, fête du 15 Aout.

66. Cf. Robb, op.cit. (n. 4), p. 523.

67. E. Guldan, Die Darstellung der Incarnation Christi im Verkündigungsbild, RQ 1968 , p. 145, fig. 19a, b et 23 b respectivement.

68. Idem, op.cit. et passim. 


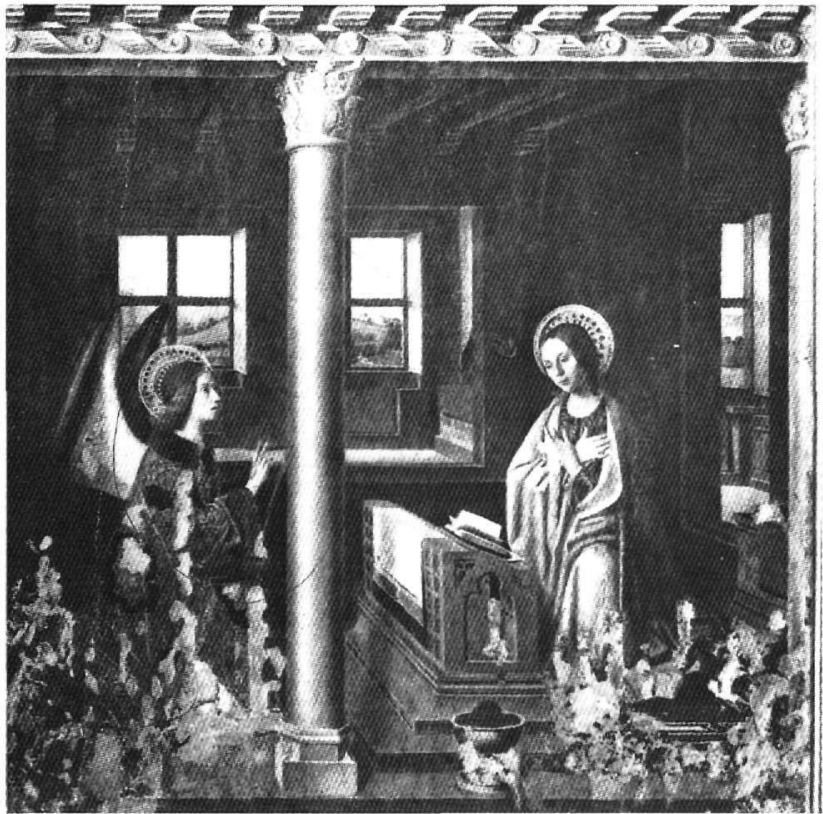

Fig. 13. Antonello da Messina, l'Annonciation.

la présence invisible du Christ au moment du message angélique, symbolise la pureté virginale de Marie et son rôle dans l'Incarnation en tant que mère et fondement de Dieu. Cette dernière nuance deviendra encore plus claire, lorsque nous examinerons le symbolisme de la colonne dans l'Annonciation byzantine (cf. infra).

\section{LA COLONNE DANS L'ANNONCIATION BYZANTINE}

Notre étude jusqu'à présent nous a montré que, dans l'art paléochrétien et du moyen âge occidental, une colonne souvent remplaçait l'image du Christ. Nous avons vu également qu'à la fin de l'époque médiévale en Occident, ce symbole s'associait au thème de l'Incarnation et par extension à la Vierge. Si dans l'art occidental le caractère symbolique de la colonne est vivant notamment dans le contexte de l'Annonciation, à Byzance, en revanche, la colonne fonctionne rarement en tant que symbole et ceci pour cause. Car, on le sait, après l'Iconoclasme, à Byzance, on représentait directement le Logos incarné et non pas son symbole. Trois questions sont ainsi posées: la signification de la colonne dans l'Annonciation byzantine, si ce motif iconographique représente un emprunt de l'art byzantin dans le répertoire occidental et, enfin, s'il s'agit d'un motif occidental, en quelle époque il est passé dans l'iconographie byzantine.

Du point de vue formel, la colonne surmontée par le

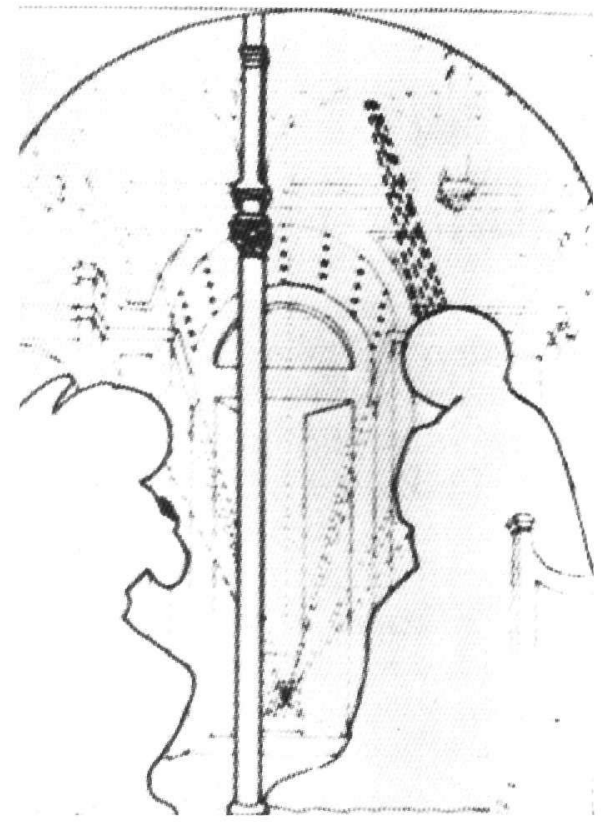

Fig. 14. Masolino da Panicale, l'Annonciation. Washington, d'après D. Arasse.

vase de fleurs est une réminiscence hellénistique. Ce fait est établi par plusieurs exemples ${ }^{69}$ (Fig. 18) et apparaît quelquefois dans des compositions de l'art byzantin, nous pensons p. ex. à une enluminure du Paris. gr. 510, dans une scène de la vie de Job ${ }^{70}$ (Fig. 19). Son apparition dans la peinture byzantine avec une valeur symbolique est parfaitement consciente, puisque le même motif se trouve dans le thème de la Présentation au temple (autre sujet de l'Incarnation), dans une icone crétoise du $\mathrm{XVe}$ siècle $^{71}$ (Fig. 20) et, évidemment, perdure dans l'Annonciation. On le rencontre dans une oeuvre de 1475, à la Galerie Tretyakov (Fig. 21). En outre, dans deux icones du XIVe siècle ${ }^{72}$, tout en faisant partie du décor architectural, le motif de la colonne se met particulièrement en|évidence, en occupant l'axe médian. Il est donc possible que sa raison d'être soit symbolique. De même, dans deux Annonciations de Patmos ${ }^{73}$, une du XVe (Fig. 22) et l'autre du XVIIe siècle, très probablement la colonne qui est placée dans la fond de la composition représente une métaphore; le vase aux fleurs, pourtant, figure au premier plan, à gauche de Marie. Enfin, dans un triptyque macédonien du XVIIe siècle ${ }^{74}$ (Fig. 23), à côté du trône de la Vierge, par manque de place la colonne coincide avec un élément du trône et apparaît surmontée par le pot aux fleurs.

En effet, un arrière plan de littérature patristique, qui loue Marie pour sa singularité dans le genre humain, en lui attribuant certains "types" bibliques ${ }^{75}$, justifie l'ap- 
parition de cet élément, en matière artistique. Le motif complexe de la colonne avec un chapiteau corinthien, au surplus surmontée par le vase empli de fleurs, en premier lieu, rappelle le bâton d'Aaron. Il se rapproche de même de l'idée relative de l'arbre et, enfin, il fait directement appel à d'autres préfigurations typologiques de la Vierge. Dans les lignes qui suivent nous examinerons les trois cas.

Le miracle du bâton fleuri, qui est associé au prophète Aaron (Num. XVII, 16-24), se rapproche de l'Incarnation et de la pureté de Marie, dans les textes théologiques. Dans une homélie d'André de Crète sur la Naissance de Marie, la Vierge est appelée: $\rho \alpha ́ \beta \delta o \varsigma$, 'A $\alpha \rho \omega \dot{\nu}$ $\dot{\alpha} \varepsilon \imath \theta \alpha \lambda \bar{\eta}^{76}$. Dans un autre texte de Theodore Stoudite

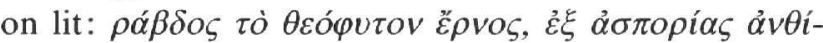
$\sigma \alpha \sigma \alpha$, et ainsi dans de nombreux exemples et des hymnes. Ainsi, au début de la 3ème ode d'un chant dit la veille de la Naissance de Marie (le 8 Septembre) on en-

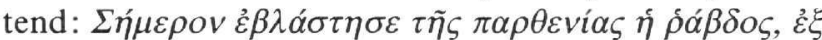

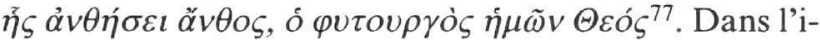
conographie, le bâton fleuri se place dans le contexte du tabernacle du témoignage et en corrélation avec l'image de la Vierge. Notamment, dans la peinture monumentale paléologue, le bâton est un attribut régulier d'Aa$\operatorname{ron}^{78}$. Le fait que le bâton fleuri est une idée proche de l'arbre est très clairement représenté dans une composition occidentale. Dans un "Speculum humanae salvationis" du XIVe siècle ${ }^{79}$, dans une scène qui représente le miracle du bâton d'Aaron, on note que parmi douze pieux dressés dans le sol, seul celui d'Aaron possède des racines et des branches, devenant ainsi un véritable arbre.

69. J. Charbonneaux, R. Martin, F. Villard, Grèce hellénistique (L'univers des formes, 18), 1970, fig. 178: Boscotrecase, peinture murale: paysage boucolique. Naples, Musée National.

70. H. Omont, Miniature des plus anciens manuscrits grecs de la Bibliothèque Nationale, du VIe au XIVe siècle, Paris 1929, pl. XXVII, fol. $71 \mathrm{v}$.

71. Cf. Baltoyannis, op.cit. (n. 1), p. 59.

72. V. Lasarev, Byzantine Icons of the XIVth and XVth Centuries, BurlMag, Decembre 1937, figs. A et B.

73. M. Chatzidakis, Icons of Patmos, Athènes 1985, pl. 81 et 86 respectivement.

74. Chr. Baltoyanni, Icons. The D. Ekonomopoulos Collection, Athènes 1986, pl. 77.

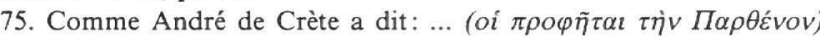

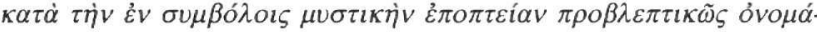

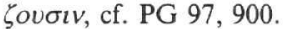

76. PG $127,609 \mathrm{C}$.

77. Mnvaĩov, Septembre.

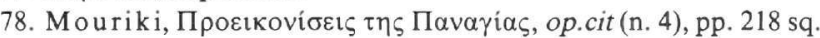
79. Speculum humanae salvationis. Described and prefaced by M. R. James, Oxford 1926, facs. of Hunterian ms of Mielot's Prose Version, chapter III.

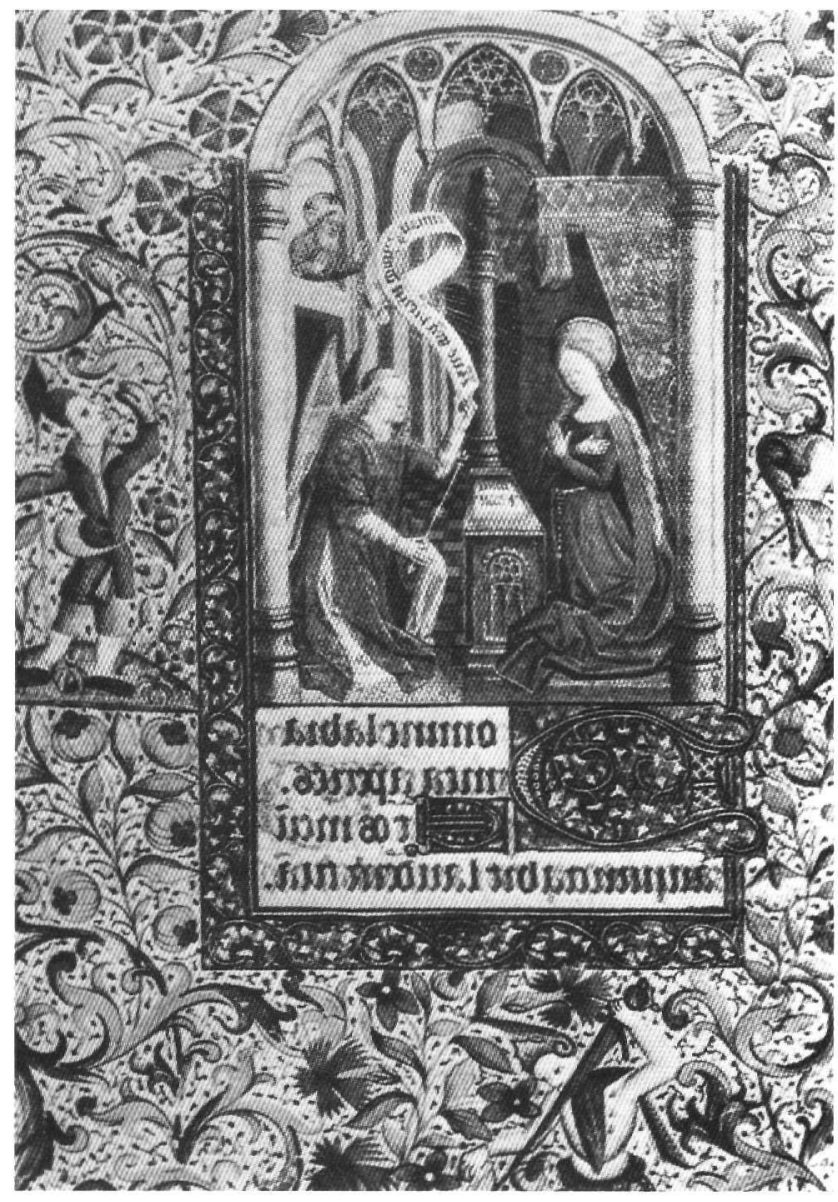

Fig. 15. Cod. Guelf. 56.8 Augusteus 40, f. 29r. L'Annonciation.

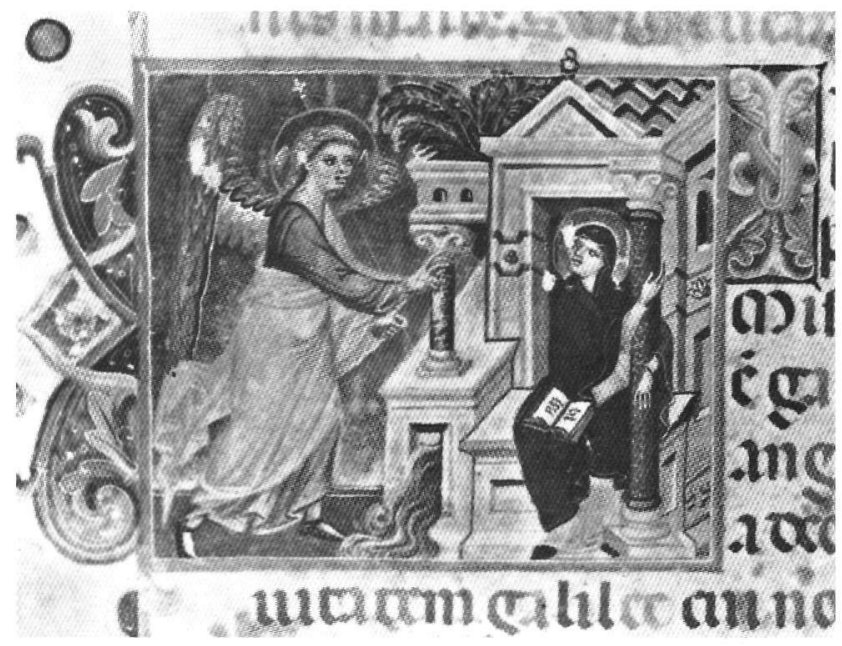

Fig. 16. Cod. lat. I, 100. L'Annonciation. 


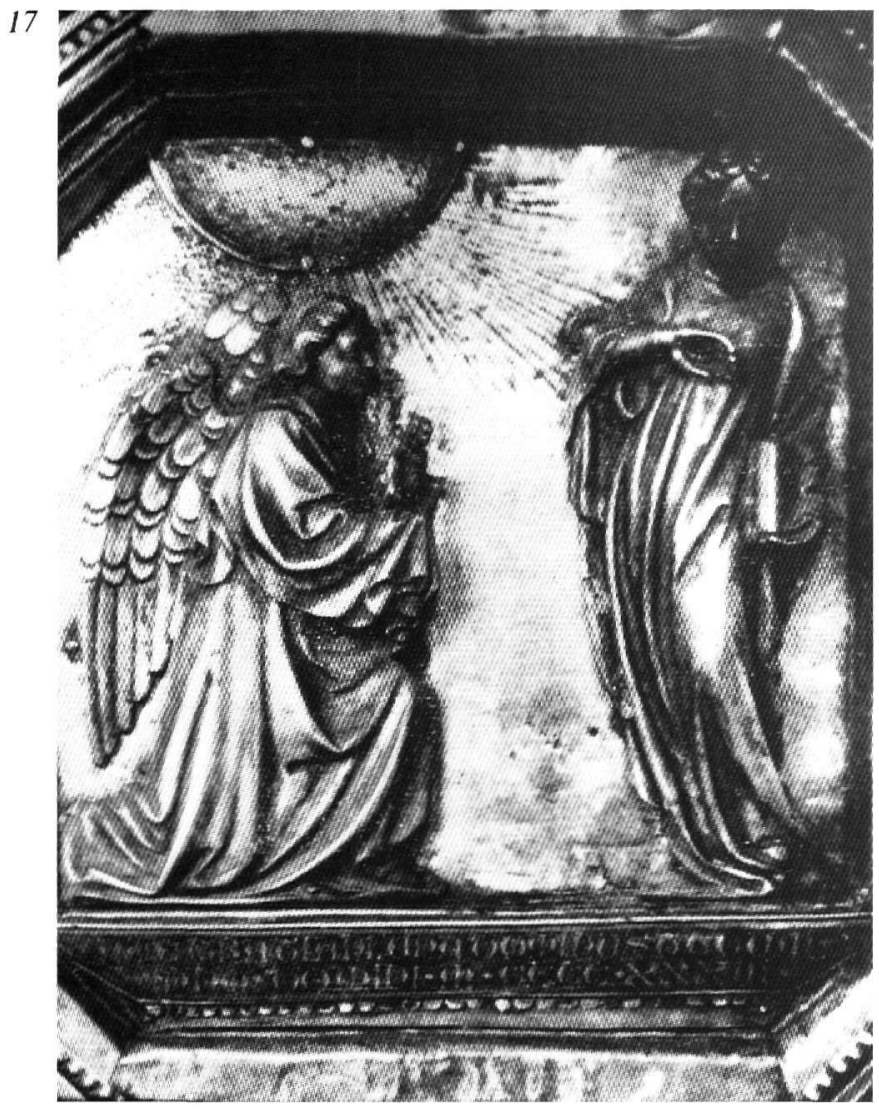

Comme il a été noté antérieurement, le rôle des métaphores littéraires, d'images souvent printanières, sur l'introduction du motif de l'arbre ou de la plante dans la scène de l'Annonciation a été très important. Dans l'Hymne Akathiste qui célèbre l'incarnation du Christ à partir de l'Annonciation jusqu'à la Présentation au temple on trouve toute une série de métaphores adressées à la Vierge: Salut, qui plantes le planteur de la vie; Salut, fleur d'immortalité; Salut, arbre de fruit magnifique, par lequel les fidèles sont nourris; Salut, arbre aux feuilles qui donnent une bonne ombre, sous lequel nombreux sont abrités... ${ }^{80}$. Dans une homélie attribuée à Jean Damascène Marie est décrite comme plus suave que le lys et plus rouge que la rose ${ }^{81}$. Léon VI dépeint Marie en tant que paradis plein de fruits, lys et roche par où sourd la fontaine de vie ${ }^{82}$. Dans un poème de Théodore Lascaris Marie est l'odeur doux des champs, la fleur...83.

Dans d'autres textes, l'idée de l'arbre ne représente plus des métaphores printanières. Dans une homélie du Ve siècle, Marie est louée en tant que bâton fleuri de Jessé, paradis de la pureté qui ne fane jamais, dans lequel l'arbre de vie est planté qui portera les fruits de la rédemption pour tous ${ }^{84}$. Jean Damascène s'adresse à
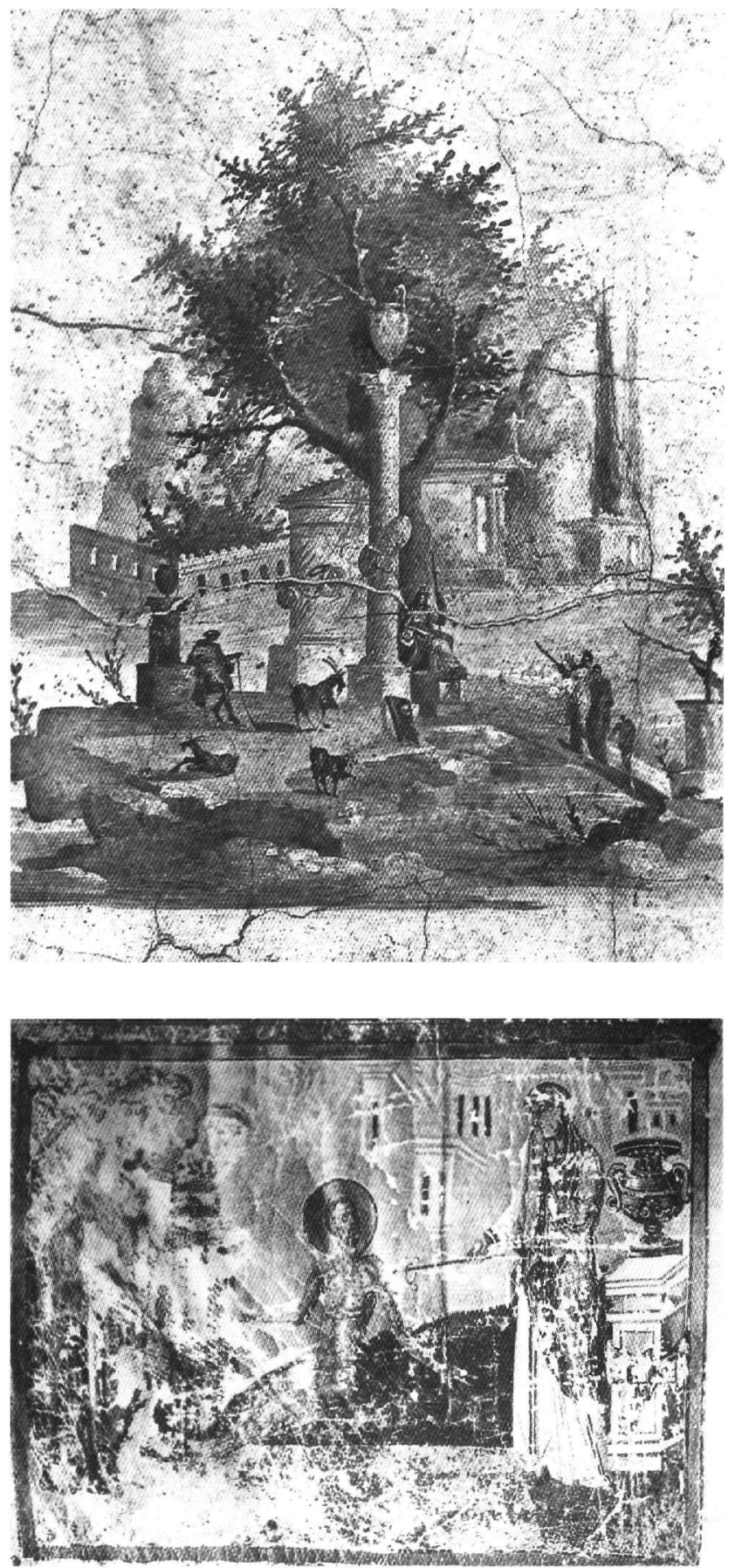

Fig. 17. Retable de Nicola da Guardagrele avec l'Annonciation.

Fig. 18. Boscotrecase, peinture murale: paysage boucolique (détail). Naples, Musée National.

Fig. 19. Cod. Paris. gr. 510, f. 71v. Détail avec une scène de la vie de $J o b$. 
Marie de façon similaire, dans une homélie sur l'Annonciation: Salut, paradis, site béate d'Edem, où toute la fleur de la vertu a fleuri et où l'arbre de vie a appa$r u^{85}$. Ailleurs, André de Crète écrit : Le paradis intelligible de l'arbre qui donne la vie et la rédemption ${ }^{86}$. Ainsi, la notion de l'arbre implique celle de l'arbre de vie, de l'arbre de Jessé, de l'arbre de la croix. Ces idées sont plutôt familières dans le contexte de l'Annonciation, puisque à la fête même du 25 Mars un chant se réfère à la crucifixion du Seigneur: 'Ev $\mu \varepsilon \dot{\varepsilon} \omega \omega \delta$ v́o $\lambda \eta \sigma \tau \tilde{\omega} v \dot{v} \psi \omega \dot{ }-$

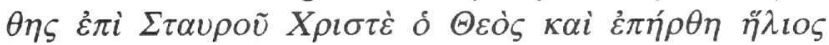

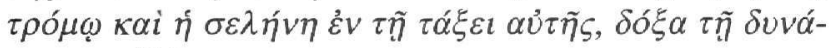

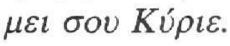

En matière artistique, très explicites sont des exemples d'Annonciations occidentales, bien que le motif de l'arbre soit un élément très courant dans l'Annonciation byzantine ${ }^{87}$. Dans un tableau des alentours de $1370-$ 1380 , partie d'un retable provenant d'une ancienne église cistercienne de Netze, entre l'ange et la Vierge de l'Annonciation, on retrouve le motif de l'arbre de Jessé (Fig. 24). La prophétie messianique d'Isaïe ${ }^{88}$ suivant laquelle de la branche poussera une fleur a été associée au contexte de l'Annonciation par la littérature latine également, ceci depuis l'époque paléochrétienne ${ }^{89}$. Presque à la même époque que ce tableau de Westfalie, un relief d'albâtre a été exécuté en Angleterre avec l'Annonciation pour thème ${ }^{90}$. Dans un vase, entre Gabriel et

20

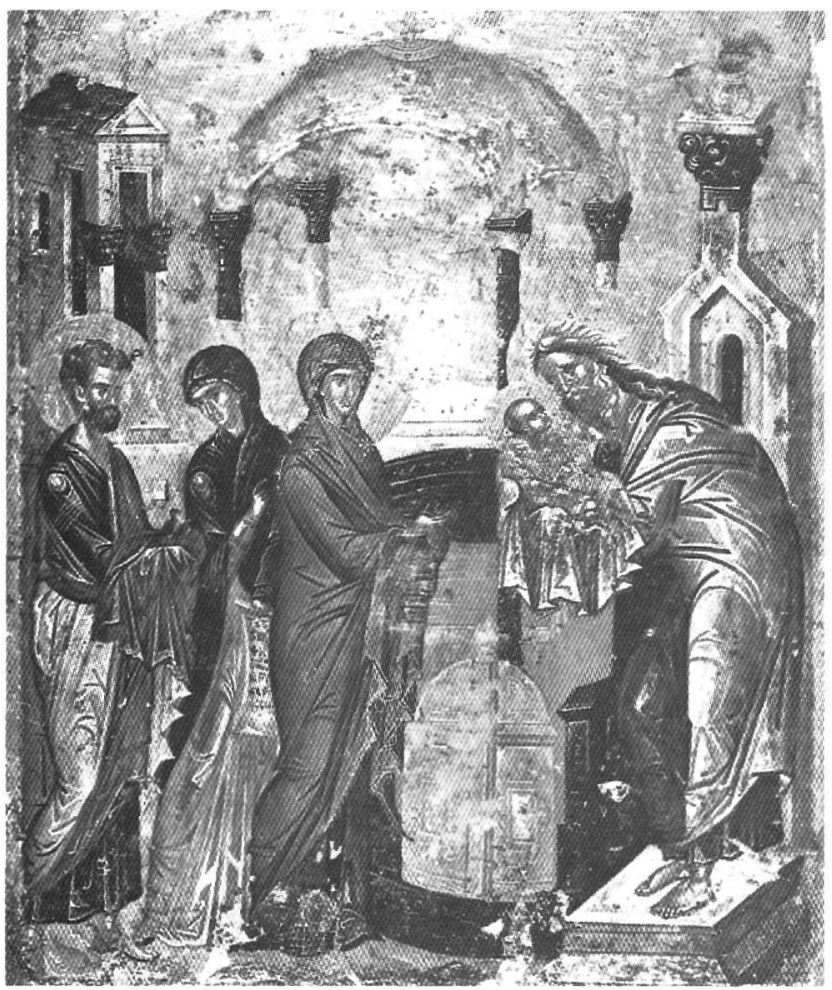

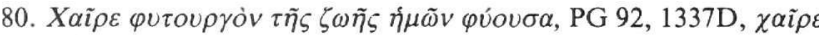

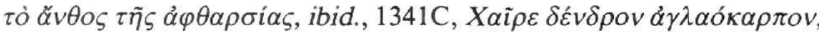

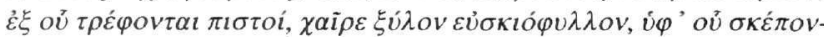
$\tau \alpha t \pi 0 \lambda \lambda o i$, ibid., 1341C-D.

81. In Annuntiationem, PG 96, 656C

82. In Annuntiationem, PG 107, 24D-25B.

83. Eustratiades, Theotokarion, pp. 39-42.

84. In Sanctissimae Deipare Annuntiationem, PG 85, 441.

85. PG 96, 713B.

86. PG $97,900$.

87. Un arbre figure déjà dans la fresque de l'Annonciation de SainteMarie de Castelseprio, VIIe siècle (G. P. Bognetti, G. Chierici et A. De Capitani D'Arzago, Santa Maria di Castelseprio, Milan 1948 , pp. 544 sq. et pl. 38a). Un ou plusieurs arbres peuvent être repérés dans l'arrière plan de certaines Annonciations byzantines du XIe siècle, comme p. ex., d'un lectionnaire du Mont Athos, Dionysiou ms 587, fol. 150; (Maguire, op.cit. (n. 10), fig. 36). A la fin du moyen âge, des exemples de ce motif sont particulièrement fréquents. P. ex., l'arbre ne manque pas dans une icone du Musée de Recklinghausen (D. and T. Talbot Rice, Icons and their Dating, Londres 1974, fig. 179), dans une icone de Temple Gallery (Byzantine Greek and Russian Icons, Exhibition 18th April to 4th August 1979, Temple Gallery, Londres, no. 29, fig. 44) ou dans une autre icone, jadis au Musée de Prague (N. M. Belajev, L'Annonciation. Monument nouveau de peinture grecque sur bois (en russe avec traduction en français), SemKond, Prague 1927, I, pp. 224 sq., pl. XX.1, 2, 3). Cf. Balt oyannis, op.cit. (n. 1), figs. $3,4,5$.

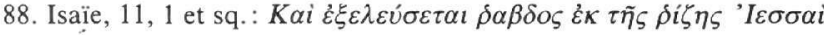

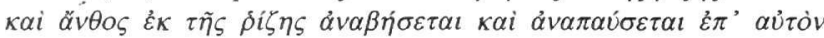
$\pi v \varepsilon \tilde{u} \mu \alpha \Theta \varepsilon \circ \tilde{u} . .$.

89. Tertullien, De carne Christi 21; Bernarnd de Clairvaux, Homilia 2 Super missus est, PL 183, 63 .

90. Guldan, op.cit. (n. 67), p. 161, pl. 28a.

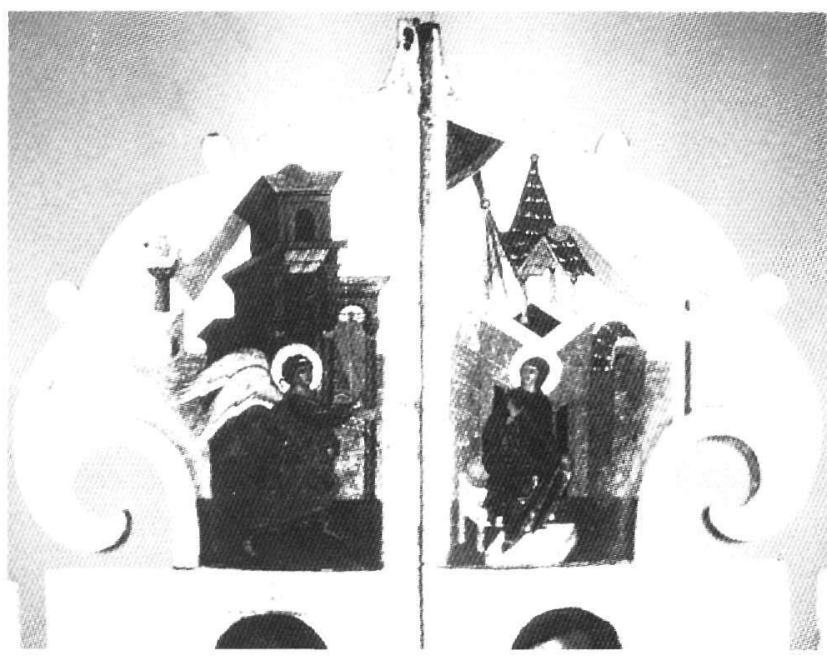

Fig. 20. Icone crétoise du XVe siècle. La Présentation au temple.

Fig. 21. Détail avec l'Annonciation sur porte d'iconostase de la Galerie Tretyakov. 

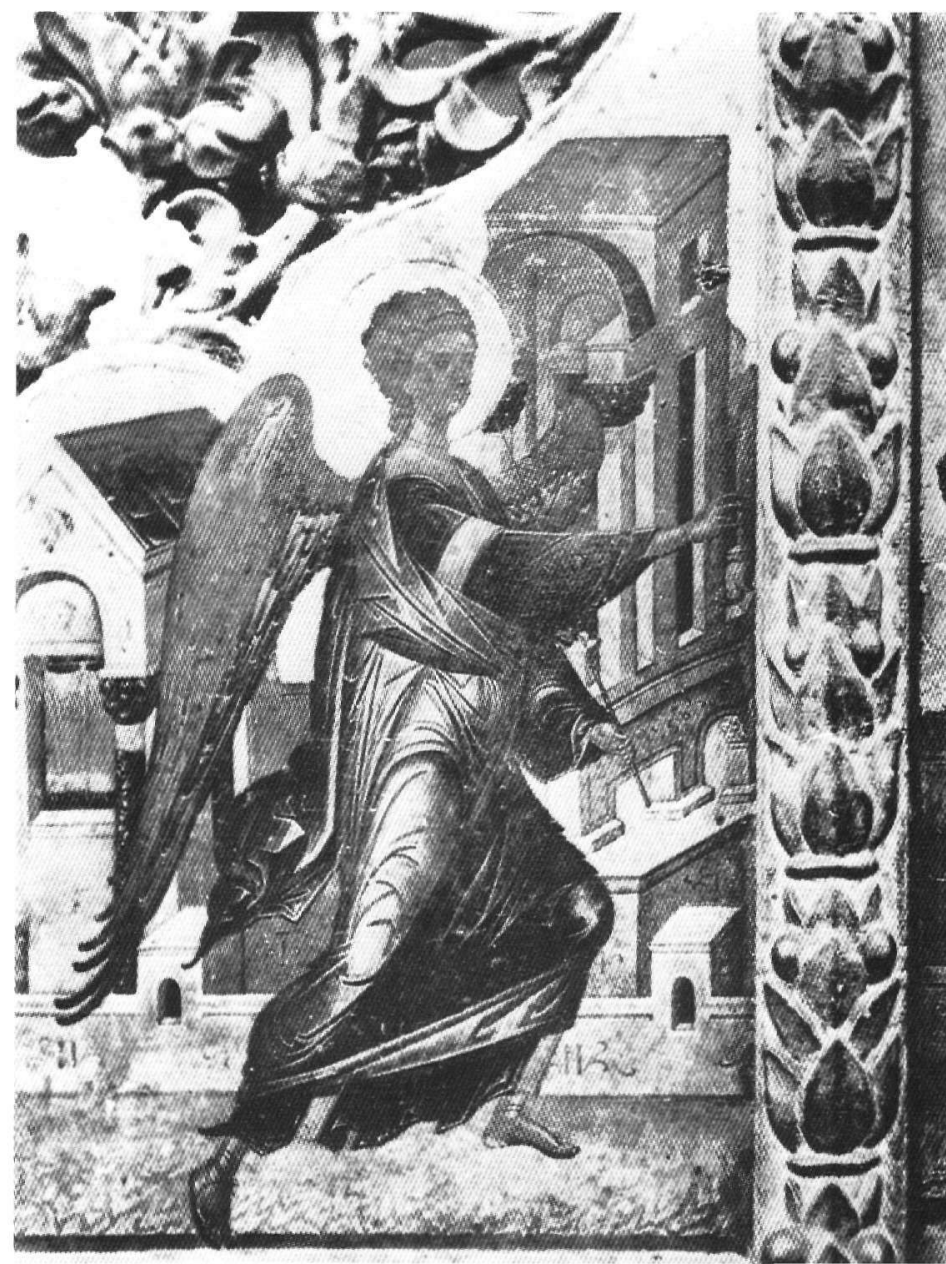

Marie, pousse une plante-arbre. Sur la partie supérieure de la composition et au sommet de cette plante est représenté le Crucifié, qui se détache devant le trône de Dieu le Père. Ce n'est plus seulement l'arbre de Jessé qui s'associe au vase de fleurs, mais aussi l'arbor crucis. Cette représentation est très riche en rapprochements d'idées qui regardent le dessein divin pour le salut humain. La scène initiale de l'Incarnation, l'Annonciation, marque également la fondation de l'Eglise sur terre, ce qui a été seulement possible à travers la passion du Christ dont le royaume éternel est représenté par la présence du Dieu le Père assis sur le trône. Le vase et l'arbre sont des types vétérotestamentaires de l'Incarnation ${ }^{91}$, dont l'instrument est la Vierge. Autrement dit, deux symboles préfigurants l'Avent et "types" de Marie, image même de l'Eglise, se juxtaposent aux événements historiques cruciaux de l'ère de la Grâce, le tout étant dominé par l'existence éternelle de Dieu. En dernière analyse, il s'agit là d'une composition de l' "Ecclesia universalis". Dans le cadre de la scène de l'Annoncia- tion, tout le dessein divin sur le salut de l'humanité est évoqué.

Enfin, l'élément de la colonne est lié aux événements décrits dans le livre de l'Exode (XIV, 19.24) ou ailleurs (Num. XIV, 14; Neem. IX, 12, 19; Sap. XVII, 3), où la présence divine se manifestait au peuple juif en tant que colonne de feu ou de nuée. Ce fait a été également interprété par les théologiens en tant que préfiguration de l'Incarnation et bien sûr de Marie. L'importance de ces chapitres vétérotestamentaires, on le sait, est très grande pour le culte marial, puisque le dernier chapitre de l'Exode, presque en entier est lu la veille du 21 Novembre, fête de la Présentation au temple de Marie ${ }^{92}$. Dans l'Hymne Akathiste, à la 2ème stance, Marie est saluée en tant que colonne de virginité, colonne de feu et nuée ${ }^{93}$, types de préfiguration de Marie, que Jean Damascène rappelle dans son homélie de la Nativité de la Vierge $^{94}$, aussi bien qu'un chant de la fête de l'Annonciation, le 25 Mars. D'ailleurs, la colonne de feu, en sa qualité de lumière, par analogie, se rapproche d'autres 
23

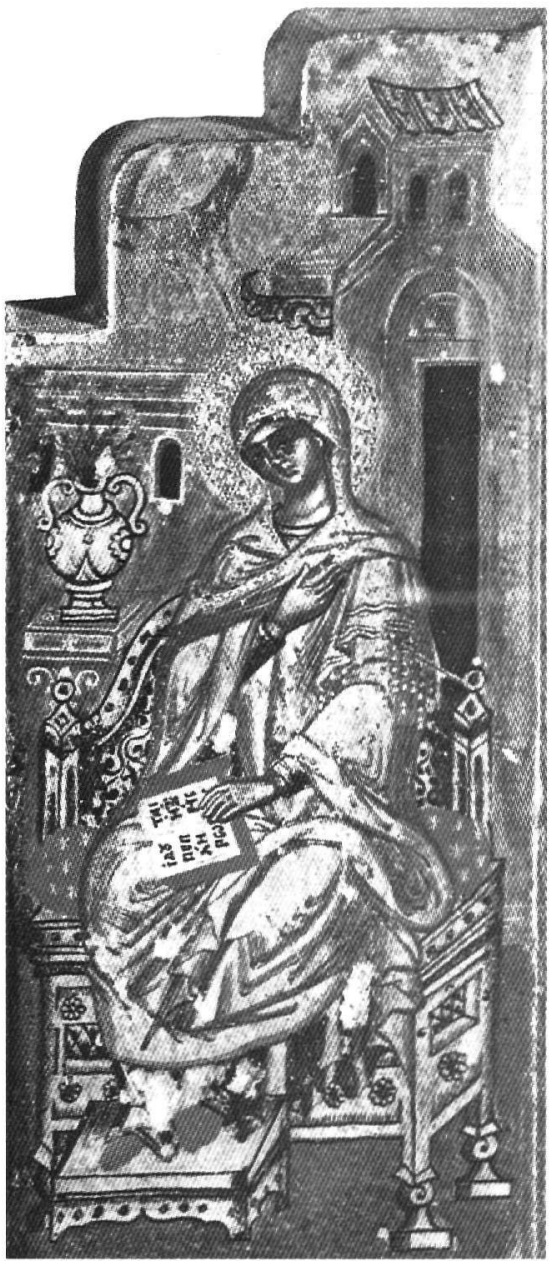

lumières-types du Christ et par extension de la Vierge. Comme Grégoire de Nazianze disait: ... la lumière en tant que feu est apparu à Moïse, lorsqu'elle brûlait le buisson, mais ne le consumait pas. La lumière en tant que colonne de feu conduisait Israel... La lumière était la beauté de l'étoile à Bethléhem qui conduisait les sages hommes...95. Ainsi Théodore Laskaris appelle Marie lumière splendide et étoile ${ }^{96}$. A la veille de la fête de l'Annonciation, dans une ode à Marie on fait appel à la nuée lumineuse ${ }^{97}$. De son côté, André de Crète, à l'occasion de la Nativité de Marie ${ }^{98}$ mentionne certains préfigurations typologiques de la Vierge en juxtaposant le chapitre biblique relatif. Entre autres, il cite la vision de feu.... émanant par la nuée... et représentant la gloire du Seigneur.

Nous arrivons donc à conclure que, dans l'Annonciation byzantine de la fin du moyen âge, le motif de la colonne représente certains "types" de préfigurations bibliques de la Vierge.

En Occident, l'usage fréquent du symbole de la colonne

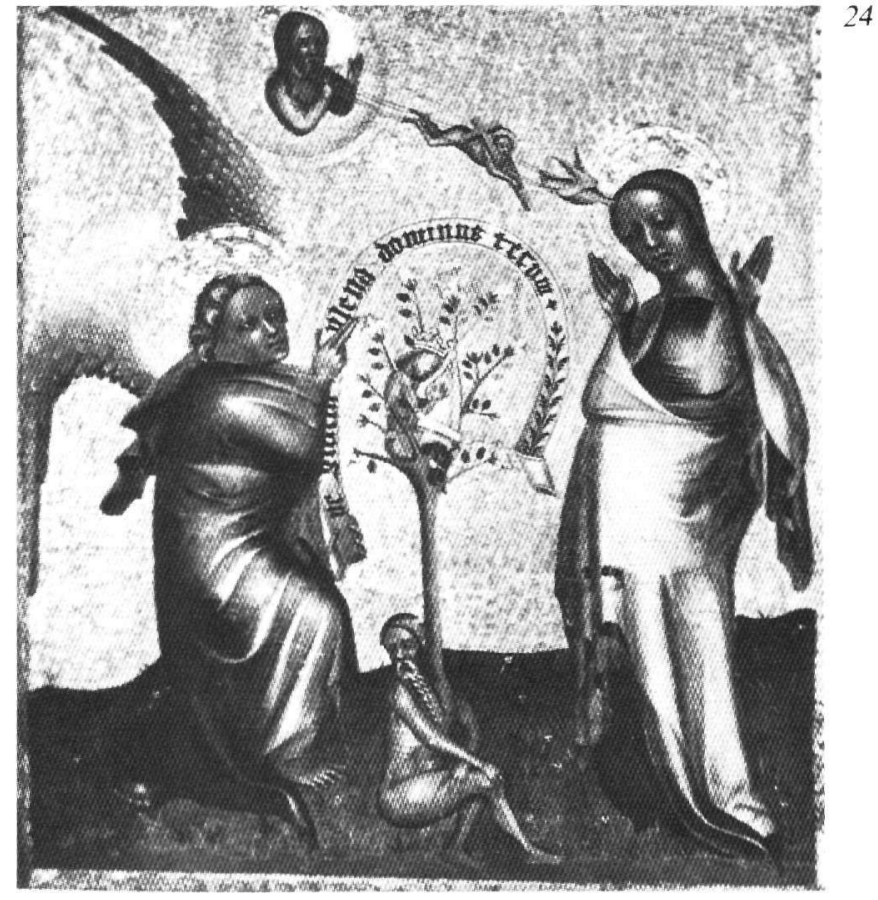

Fig. 22. Détail avec l'Annonciation sur porte d'iconostase de l'école crétoise du XVe siècle, provenant de Patmos.

Fig. 23. Détail avec la Vierge de l'Annonciation sur un triptyque macédonien du XVIIe siècle.

Fig. 24. Retable de Netze.

91. En ce qui concerne l'arbre, entre autres cf. au psaume 1, 3 où le Christ se substitue à l'arbre planté près de la source de l'eau qui

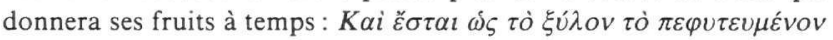

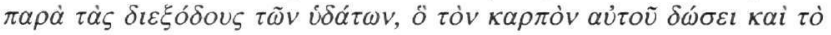

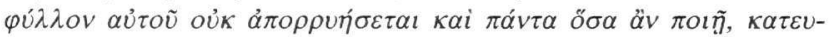

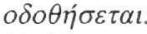

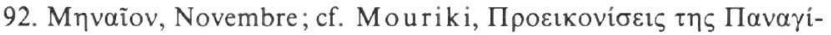
a与, op.cit. (n. 4), p. 225; G. Babić, Image symbolique de la 'porte fermée' à Saint-Clément d'Ohrid, Synthronon (Bibliothèque des Cahiers Archéologiques II), Paris 1968, pp. 146-151.

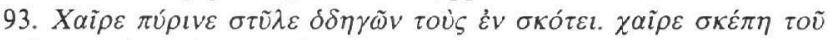

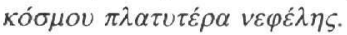

94. PG $96,673 \mathrm{~B}$ et $696 \mathrm{~A}$.

95. PG 36, 364D-365B.

96. Eustratiades, Theotokarion, pp. 39-42.

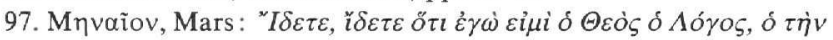

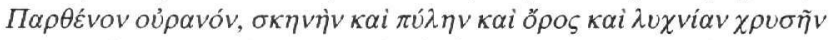

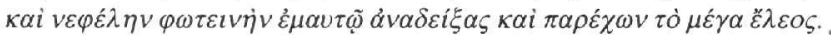

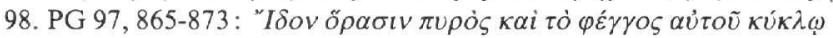

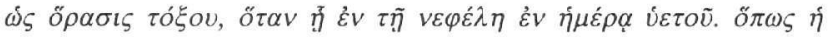

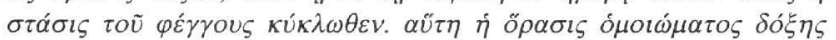
Kupiou. 
témoigne que les Byzantins ont sans doute puisé dans le répertoire de l'art occidental. Ce motif doit être passé dans l'iconographie byzantine à la fin du moyen âge, où il a reçu une forme d'origine hellénistique et où son symbolisme s'attache exclusivement à Marie. La Théotokos a une place prépondérante dans la vie liturgique et spirituelle de l'Eglise Orthodoxe. Son rôle de médiatrice y est affirmé avec insistance. Par ailleurs, comme les textes le montrent en corrélation avec les exemples en matière artistique, dans les Annonciations occidentales, également, l'accent est mis sur la représentation de la colonne en tant que symbole mystique de la Mère de Dieu, sans laquelle l'Incarnation n'est pas possible. Il convient de rappeler que des hymnes comme l'Akathiste et que les homélies de théologiens grecs importants étaient traduits en latin et utilisés dans la liturgie. A la Dormition de la Vierge p. ex., le 15 Août, en l'église romaine on lit un sermon de Jean Damascène ${ }^{99}$. A la fin du moyen âge, à Byzance, l'apparition d'un symbole de l'Incarnation associé à la Vierge est parfaitement justifiée, puisque, on le sait, à Constantinople, les idées de tendences humanistes exprimaient avec force l'espoir du salut humain à travers la première Venue du Christ. Le fait que ce motif apparaît dans la fresque de l'église de Péribleptos de Mistra, dont l'iconographie exceptionnelle est connue en ce qui concerne les symboles de l'Incarnation, corrobore nos arguments.

\section{CONCLUSIONS}

Dans notre étude nous avons essayé de comprendre le message transmis par le motif de la colonne dans la scène de l'Annonciation. En examinant diverses oeuvres paléochrétiennes et occidentales nous avons vu qu'une colonne remplaçait souvent l'image du Christ ou de l'Eglise. A la fin du moyen âge occidental, ce symbole se liait, également, au thème de l'Incarnation et par extension à la Vierge.

L'intense présense du caractère vivant de la colonne dans l'art occidental représente sans doute un argument pour conclure que ce motif est apparu d'abord dans l'Annonciation occidentale, à la fin du moyen âge, en tant que symbole à la fois du Christ incarné au moment du message angélique et de la pureté virginale de Marie. De l'art occidental, ce motif est ensuite passé dans l'Annonciation byzantine de l'époque des Paléologues. Le remplacement de l'image du Christ par une colonne était inadmissible à Byzance, où après le Triomphe de l'Orthodoxie, on représentait directement le Logos incarné et non pas son symbole. Dans l'Annonciation byzantine, donc, la colonne représente exclusivement certains "types" de préfiguration bibliques de la Vierge. L'emprunt de l'art byzantin dans le répertoire occidental d'un symbole d'origine paléochrétienne, qui se liait à l'Incarnation, a été d'autant plus facilité qu'à cette époque, à Constantinople, les tendences théologiques et spirituelles humanistes accentuaient l'idée du salut humain à travers le Logos fait homme. A l'origine, à Byzance, son apparition fut très discrète (nous pensons p. ex. aux icones paléologues). Par la suite, il a reçu une forme qui correspondait tout à fait à la tradition byzantine, qui avait donc son origine dans l'art hellénistique.

Dans les pages précédentes, nous avons interprété le motif iconographique de la colonne en tant que symbole dans certaines compositions de l'Annonciation. Il se lie au thème de l'Incarnation et devient un attribut de la Vierge. Marie est une colonne, car cet élément ressemble à un arbre, comme l'arbre de Jessé, le bâton fleuri d'Aaron, l'arbre de vie ou l'arbre de la croix. Elle est une colonne, car Dieu se révélait en tant que colonne de nuée ou de feu. Elle est une colonne, car la colonne de feu-lumière se rapproche de toutes les lumières qui préfigurent la Vierge. Enfin, elle est la colonne de virginité, le fondement du Christ et l'Eglise. Grâce à cette interprétation, nous avons eu l'occasion de constater la richesse idéologique et l'importance magistrale de la fête de la Salutation angélique, moment crucial pour le salut de l'humanité, où toute l'oeuvre de l'économie divine est évoquée.

99. Breviarium Romanum, 15 Août. 